\title{
COHERENT TANGENT BUNDLES AND GAUSS-BONNET FORMULAS FOR WAVE FRONTS
}

\author{
KENTARO SAJI, MASAAKI UMEHARA, AND KOTARO YAMADA \\ Dedicated to Professor Toshiki Mabuchi on the occasion of his sixtieth birthday
}

\begin{abstract}
We give a definition of 'coherent tangent bundles', which is an intrinsic formulation of wave fronts. In our application of coherent tangent bundles for wave fronts, the first fundamental forms and the third fundamental forms are considered as induced metrics of certain homomorphisms between vector bundles. They satisfy the completely same conditions, and so can reverse roles with each other. For a given wave front of a 2-manifold, there are two Gauss-Bonnet formulas. By exchanging the roles of the fundamental forms, we get two new additional Gauss-Bonnet formulas for the third fundamental form. Surprisingly, these are different from those for the first fundamental form, and using these four formulas, we get several new results on the topology and geometry of wave fronts.
\end{abstract}

\section{Introduction}

In this paper, we give a definition of coherent tangent bundles, which is an intrinsic formulation of wave fronts. The advantage of this formulation is that the first fundamental forms and the third fundamental forms satisfy exactly the same conditions for wave fronts in space forms, and they can reverse roles with each other. Then the two Gauss-Bonnet formulas induce two more Gauss-Bonnet formulas by exchanging the first and third fundamental forms. These turn out to be different from those for the first fundamental form, and using these four formulas, we get new results on fronts.

In Section 2, we generalize the definitions of coherent tangent bundles and singular curvature, which were given for 2-dimensional coherent tangent bundles (or fronts) in [13] and 14, to fronts of general dimension. A coherent tangent bundle on an $m$-manifold $M^{m}$ induces a positive semi-definite metric on $M^{m}$ (called the first fundamental form), and can be regarded as a generalization of Riemannian manifolds. Using the concept of coherent tangent bundles, we can give a unified treatment of hypersurfaces and $C^{\infty}$-maps between the same dimensional manifolds. The points where the metric degenerates are called singular points. We define a notion of $A_{k}$ (singular) points, which is a generalization of that of fronts and of $C^{\infty}$-Morin maps at the same time. We define singular principal curvatures for each $A_{2}$-point of a given coherent tangent bundle, which are $(m-1)$-tuples of real

Date: November 6, 2010.

2000 Mathematics Subject Classification. Primary 57R45; Secondary 53A05.

Key words and phrases. wave front, curvature, the Gauss-Bonnet formula, fold.

K. Saji, M. Umehara and K. Yamada were partially supported by Grant-in-Aid for Scientific Research (Young Scientists (B)) No. 20740028, (A) No.22244006 and (B) No. 21340016, respectively from the Japan Society for the Promotion of Science. 
numbers. One of them diverges to $-\infty$ at $A_{3}$-points as shown in Theorem 2.12 This is a generalization of the result in [13] for 2-dimensional fronts. Moreover, as shown in Section 3 , our intrinsic setting enables us to introduce the singular curvature on $A_{2}$-Morin singular points. When $m=2$, as an application of the two intrinsic Gauss-Bonnet formulas, we give a new proof of Quine's formula (cf. Proposition 3.6), and also get a new formula (cf. Proposition 3.7) for the total singular curvature of generic smooth maps between 2-manifolds.

Furthermore, in Section 3, we also give several results on compact 2-dimensional fronts, as an application of our new Gauss-Bonnet formulas for the third fundamental form. In particular, we show that the total negative Gaussian curvature $\int_{M^{2}} \min (0, K) d A$ of a closed immersed surface $M^{2}$ in $\boldsymbol{R}^{3}$ is equal to the signed sum of total geodesic curvature on each connected component of the singular set of its Gauss map, see Theorem 3.18. The deepest applications are given for surfaces of bounded Gaussian curvature. For example, the Euler characteristic of a closed wave front with Gaussian curvature $-1 / \delta<K<-\delta(\delta>0)$ in a 3 -dimensional flat torus vanishes (cf. Theorem 3.30).

\section{Coherent tangent Bundles}

Let $M^{m}$ be an oriented $m$-manifold $(m \geq 1)$ and $\mathcal{E}$ a vector bundle of rank $m$ over $M^{m}$. Before defining coherent tangent bundles, we define $A_{k}$-points for each vector bundle homomorphism from the tangent bundle of $M^{m}$ to $\mathcal{E}$.

2.1. $A_{k}$-points. We assume that $\mathcal{E}$ is orientable, namely there exists a non-vanishing section $\mu: M^{m} \rightarrow \operatorname{det}\left(\mathcal{E}^{*}\right)$, where $\operatorname{det}\left(\mathcal{E}^{*}\right)$ is the determinant line bundle of the dual bundle $\mathcal{E}^{*}$. This assumption does not create any restriction for the local theory. In fact, for each point $p \in M^{m}$, there exists a section $\mu$ of $\operatorname{det}\left(\mathcal{E}^{*}\right) \backslash\{0\}$ defined on a neighborhood $U$ of $p$. Now we fix a bundle homomorphism

$$
\varphi: T M^{m} \longrightarrow \mathcal{E}
$$

where $T M^{m}$ is the tangent bundle of $M^{m}$. A point $p \in M^{m}$ is called a $\varphi$-singular point if $\varphi_{p}: T_{p} M^{m} \rightarrow \mathcal{E}_{p}$ is not a bijection, where $\mathcal{E}_{p}$ is the fiber of $\mathcal{E}$ at $p$. We denote by $\Sigma_{\varphi}$ the set of $\varphi$-singular points on $M^{m}$. On the other hand, a point $p \in M^{m} \backslash \Sigma_{\varphi}$ is called a $\varphi$-regular point. We now fix a $\varphi$-singular point $p$, and take a positively oriented local coordinate neighborhood $\left(U ; u_{1}, \ldots, u_{m}\right)$ of $p$. THen it holds that

$$
\varphi^{*} \mu=\lambda_{\varphi} d u_{1} \wedge \cdots \wedge d u_{m}
$$

where

$$
\lambda_{\varphi}=\mu\left(\varphi_{1}, \ldots, \varphi_{n}\right): U \rightarrow \boldsymbol{R}, \quad\left(\varphi_{j}=\varphi\left(\frac{\partial}{\partial u_{j}}\right), j=1, \ldots, m\right)
$$

is a $C^{\infty}$-function. We call the function $\lambda_{\varphi}$ the $\varphi$-Jacobian function. The set of $\varphi$-singular points on $U$ is expressed as

$$
\Sigma_{\varphi} \cap U:=\left\{p \in U ; \lambda_{\varphi}(p)=0\right\} .
$$

We now set

$$
M_{\varphi}^{+}:=\left\{q \in M^{m} ; \lambda_{\varphi}(q)>0\right\}, \quad M_{\varphi}^{-}:=\left\{q \in M^{m} ; \lambda_{\varphi}(q)<0\right\} .
$$

Since the sign of $\lambda_{\varphi}$ does not depend on the choice of the positively oriented local coordinate system, the above definitions of $M_{\varphi}^{ \pm}$make sense. 
A $\varphi$-singular point $p\left(\in \Sigma_{\varphi}\right)$ is called non-degenerate if $d \lambda_{\varphi}$ does not vanish at $p$. On a neighborhood of a non-degenerate $\varphi$-singular point, the $\varphi$-singular set consists of an $(m-1)$-submanifold in $M^{m}$, called the $\varphi$-singular submanifold. If $p$ is a non-degenerate $\varphi$-singular point, the rank of $\varphi_{p}$ is $m-1$. The direction of the kernel of $\varphi_{p}$ is called the null direction. Let $\eta$ be a null vector field, namely, smooth (non-vanishing) vector field along the $\varphi$-singular submanifold $\Sigma_{\varphi}$, which gives the null direction at each point in $\Sigma_{\varphi}$.

Definition 2.1 ( $A_{2}$-points). A non-degenerate $\varphi$-singular point $p \in M^{m}$ is called an $A_{2}$-point, or an $A_{2}$-point of $\varphi$, if the null direction $\eta(p)$ is transversal to the $\varphi$-singular submanifold.

We set

$$
\lambda_{\varphi}^{\prime}:=d \lambda(\tilde{\eta}),
$$

where $\tilde{\eta}$ is a vector field on a neighborhood $U$ of $p$ which gives a null vector field on $\Sigma_{\varphi} \cap U$. Then $p$ is an $A_{2}$-point if and only if the function $\lambda_{\varphi}^{\prime}$ does not vanish at $p$ (see [16, Theorem 2.4]).

Definition 2.2 ( $A_{3}$-points). A non-degenerate $\varphi$-singular point $p \in M^{m}$ is called an $A_{3}$-point or an $A_{3}$-point of $\varphi$ if it is not an $A_{2}$-point of $\varphi$, but $d \lambda_{\varphi}^{\prime}(\eta)$ does not vanish.

Similarly, we can define $A_{k+1}$-points inductively. We set

$$
\lambda_{\varphi}^{(k)}:=d \lambda_{\varphi}^{(k-1)}(\tilde{\eta}): \Sigma_{\varphi} \rightarrow \boldsymbol{R} \quad\left(\lambda_{\varphi}^{(1)}:=\lambda_{\varphi}^{\prime}, \lambda_{\varphi}^{(0)}:=\lambda_{\varphi}\right) .
$$

If $\left(d \lambda_{\varphi}^{(k-1)}\right)_{p} \neq 0$ on $T_{p} \Sigma^{k-1}$, then we can define a subset of the submanifold $\Sigma_{\varphi}^{k}$ by

$$
\Sigma_{\varphi}^{k+1}:=\left\{q \in \Sigma_{\varphi}^{k} ; \lambda_{\varphi}^{(k)}(q)=0\right\}=\left\{q \in \Sigma_{\varphi}^{k} ; \eta(q) \in T_{q} \Sigma_{\varphi}^{k}\right\}
$$

where we set $\Sigma_{\varphi}^{1}:=\Sigma_{\varphi}$ and $\Sigma_{\varphi}^{0}:=M^{m}$. If a non-degenerate $\varphi$-singular point $p$ is not an $A_{k}$-point of $\varphi$, but $d \lambda_{\varphi}^{(k-1)}(\tilde{\eta})$ is well-defined inductively and is nonvanishing at $p$, then it is called an $A_{k+1}$-point of $\varphi$. Here an $A_{1}$-point means a $\varphi$-regular point. (See [17, Section 2] for details.)

2.2. Coherent tangent bundles. As a generalization of the 2-dimensional case in 13 and [14, we give a general setting for intrinsic fronts: Let $M^{m}$ be an oriented $m$-manifold $(m \geq 1)$. A coherent tangent bundle over $M^{m}$ is a 5 -tuple $\left(M^{m}, \mathcal{E},\langle\rangle, D,, \varphi\right)$, where

(1) $\mathcal{E}$ is a vector bundle of rank $m$ over $M^{m}$ with an inner product $\langle$,$\rangle ,$

(2) $D$ is a metric connection on $(\mathcal{E},\langle\rangle$,$) ,$

(3) $\varphi: T M^{m} \rightarrow \mathcal{E}$ is a bundle homomorphism which satisfies

$$
D_{X} \varphi(Y)-D_{Y} \varphi(X)-\varphi([X, Y])=0
$$

for vector fields $X$ and $Y$ on $M^{m}$.

In this setting, the pull-back of the metric

$$
d s_{\varphi}^{2}:=\varphi^{*}\langle,\rangle
$$

is called the $\varphi$-metric, which is a positive semidefinite symmetric tensor on $M^{m}$.

It should be remarked that as in (2.6),

$$
T_{\varphi}(X, Y):=D_{X} \varphi(Y)-D_{Y} \varphi(X)-\varphi([X, Y])
$$


gives a skew-symmetric tensor on $M^{m}$, called the torsion tensor of $D$ with respect to $\varphi$. If $\left(M^{m}, \mathcal{E},\langle\rangle, D,, \varphi\right)$ is a coherent tangent bundle, then the pull back of the connection $D$ by $\varphi$ coincides with the Levi-Civita connection. In this sense, a coherent tangent bundle can be considered as a generalization of Riemannian manifold. In fact, as an application, a duality of conformally flat manifolds with admissible singularities is given in terms of coherent tangent bundles in 9 .

A coherent tangent bundle $\left(M^{m}, \mathcal{E},\langle\rangle, D,, \varphi\right)$ is called co-orientable if the vector bundle $\mathcal{E}$ is orientable, namely, there exists a globally defined smooth section $\mu$ of $\operatorname{det}\left(\mathcal{E}^{*}\right)$ such that

$$
\mu\left(\boldsymbol{e}_{1}, \ldots, \boldsymbol{e}_{m}\right)= \pm 1
$$

for any orthonormal frame $\left\{\boldsymbol{e}_{1}, \ldots, \boldsymbol{e}_{m}\right\}$ on $\mathcal{E}$. The form $\mu$ is determined uniquely up to a \pm -ambiguity. A co-orientation of the coherent tangent bundle $\mathcal{E}$ is a choice of $\mu$. An orthonormal frame $\left\{\boldsymbol{e}_{1}, \ldots, \boldsymbol{e}_{m}\right\}$ is called positive with respect to the co-orientation $\mu$ if $\mu\left(\boldsymbol{e}_{1}, \ldots, \boldsymbol{e}_{m}\right)=+1$.

We give here typical examples of coherent tangent bundles:

Example 2.3. Let $M^{m}$ be an oriented $m$-manifold and $\left(N^{m}, g\right)$ an oriented Riemannian $m$-manifold. A $C^{\infty}$-map $f: M^{m} \rightarrow N^{m}$ induces a coherent tangent bundle over $M^{m}$ as follows: Let $\mathcal{E}_{f}:=f^{*} T N^{m}$ be the pull-back of the tangent bundle $T N^{m}$ by $f$. Then $g$ induces a positive definite metric $\langle$,$\rangle on \mathcal{E}_{f}$, and the restriction $D$ of the Levi-Civita connection of $g$ gives a connection on $\mathcal{E}$ which is compatible with respect to the metric $\langle$,$\rangle . We set \varphi_{f}:=d f: T M^{m} \rightarrow \mathcal{E}_{f}$, which gives the structure of the coherent tangent bundle on $M^{m}$. A necessary and sufficient condition for a given coherent tangent bundle over an $m$-manifold to be realized as a smooth map into the $m$-dimensional space form is given in [18.

Example 2.4. Let $\left(N^{m+1}, g\right)$ be an $(m+1)$-dimensional Riemannian manifold. A $C^{\infty}$-map $f: M^{m} \rightarrow N^{m+1}$ is called a frontal if for each $p \in M^{m}$, there exists a neighborhood $U$ of $p$ and a unit vector field $\nu$ along $f$ defined on $U$ such that $g(d f(X), \nu)=0$ holds for any vector field $X$ on $U$ (that is, $\nu$ is a unit normal vector field), and the map $\nu: U \rightarrow T_{1} N^{m+1}$ is a $C^{\infty}$-map, where $T_{1} N^{m+1}$ is the unit tangent bundle of $N^{m+1}$. Moreover, if $\nu$ can be taken to be an immersion as a smooth section of $T_{1} N^{m+1}$ for each $p \in M^{m}, f$ is called a front or a wave front. We remark that $f$ is a front if and only if $f$ has a lift $L_{f}: M^{m} \rightarrow P\left(T^{*} N^{m+1}\right)$ as a Legendrian immersion, where $P\left(T^{*} N^{m+1}\right)$ is a projectified cotangent bundle on $N^{m+1}$ with the canonical contact structure. The subbundle $\mathcal{E}_{f}$ which consists of the vectors in the pull-back bundle $f^{*} T N^{m+1}$ perpendicular to $\nu$ gives a coherent tangent bundle. In fact, $\varphi_{f}: T M^{m} \ni X \mapsto d f(X) \in \mathcal{E}_{f}$ gives a bundle homomorphism. Let $\nabla$ be the Levi-Civita connection on $N^{m+1}$. Then by taking the tangential part of $\nabla$, it induces a connection $D$ on $\mathcal{E}_{f}$ satisfying (2.6). Let $\langle$,$\rangle be a metric on \mathcal{E}_{f}$ induced from the Riemannian metric on $N^{m+1}$, then $D$ is a metric connection on $\mathcal{E}_{f}$. Thus we get a coherent tangent bundle $\left(M^{m}, \mathcal{E}_{f},\langle\rangle, D,, \varphi_{f}\right)$. Since the unit tangent bundle can be canonically identified with unit cotangent bundle, the map $\nu: U \rightarrow T_{1} N^{m+1}$ can be identified with $\left.L_{f}\right|_{U}$. A frontal $f$ is called co-orientable if there is a unit normal vector field $\nu$ globally defined on $M^{m}$. When $N^{m+1}$ is orientable, the coherent tangent bundle is co-orientable if and only if so is $f$.

From now on, we assume that $\left(M^{m}, \mathcal{E},\langle\rangle, D,, \varphi\right)$ is a co-orientable coherent tangent bundle, and fix a co-orientation $\mu$ on the coherent tangent bundle. (If $\mathcal{E}$ 
is not co-orientable, one can take a double cover $\pi: \widehat{M}^{m} \rightarrow M^{m}$ such that the pull-back of $\mathcal{E}$ by $\pi$ is a co-orientable coherent tangent bundle over $\widehat{M}^{m}$.)

Definition 2.5. The signed $\varphi$-volume form $d \hat{A}_{\varphi}$ and the (unsigned) $\varphi$-volume form $d A_{\varphi}$ are defined as

$$
d \hat{A}_{\varphi}:=\varphi^{*} \mu=\lambda_{\varphi} d u_{1} \wedge \cdots \wedge d u_{m}, \quad d A_{\varphi}:=\left|\lambda_{\varphi}\right| d u_{1} \wedge \cdots \wedge d u_{m},
$$

where $\left(U ; u_{1}, \ldots, u_{m}\right)$ is a local coordinate system of $M^{m}$ compatible with the orientation of $M^{m}$, and $\lambda_{\varphi}$ is the $\varphi$-Jacobian function on $U$ given in (2.1). Both $d \hat{A}_{\varphi}$ and $d A_{\varphi}$ are independent of the choice of a positively oriented local coordinate system $\left(U ; u_{1}, \ldots, u_{m}\right)$, and give two globally defined $m$-forms on $M^{m} \cdot\left(d \hat{A}_{\varphi}\right.$ is $C^{\infty}$ differentiable, but $d A_{\varphi}$ is only continuous.) When $M^{m}$ has no $\varphi$-singular points, the two forms coincide up to sign. Then the two sets $M_{\varphi}^{ \pm}$as in (2.3) are written as

$$
\begin{aligned}
& M_{\varphi}^{+}:=\left\{p \in M^{m} \backslash \Sigma_{\varphi} ; d \hat{A}_{\varphi}(p)=d A_{\varphi}(p)\right\}, \\
& M_{\varphi}^{-}:=\left\{p \in M^{m} \backslash \Sigma_{\varphi} ; d \hat{A}_{\varphi}(p)=-d A_{\varphi}(p)\right\} .
\end{aligned}
$$

The $\varphi$-singular set $\Sigma_{\varphi}$ coincides with the boundary $\partial M_{\varphi}^{+}=\partial M_{\varphi}^{-}$.

\subsection{Singularities of 2-dimensional coherent tangent bundle.}

When $m=2$ and $\left(M^{2}, \mathcal{E},\langle\rangle, D,, \varphi\right)$ comes from a front in 3-manifold as in Example 2.4 (resp. a map into 2-manifold as in Example 2.3), an $A_{2}$-point corresponds to a cuspidal edge (resp. a fold) (cf. 15, see Fig. 11). Though cuspidal cross caps in surfaces in $\boldsymbol{R}^{3}$ are not singular points of a front, they are also $A_{2}$-points (see [14, Remark 1.6]). In this way, our definition of $A_{2}$-points are wider than the original definition of $A_{2}$-points on fronts or Morin maps. However, if $\left(M^{2}, \mathcal{E},\langle\rangle, D,, \varphi\right)$ is associated to a wave front, $A_{2}$-points really corresponds to cuspidal edges on its realization as fronts.
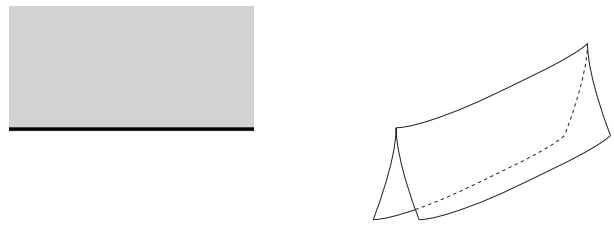

Figure 1. A fold in a plane (left) and a cuspidal edge in a space (right).

On the other hand, an $A_{3}$-point corresponds to a swallowtail (resp. a cusp) when $m=2$ and $\left(M^{2}, \mathcal{E},\langle\rangle, D,, \varphi\right)$ comes from a front in a 3-manifold (resp. a map into a 2-manifold). This fact was shown in [7 (see Fig. 2).

When $\varphi$ is induced from a smooth map between 2-manifolds (resp. a front in a 3 -manifold), it is well-known that $A_{2}$-points and $A_{3}$-points are generic singular points of $\varphi$ (cf. 1]). However, if one consider deformations of $A_{2}$ or $A_{3}$-points, an additional three types of singular points of type $A_{3}^{+}$(lips), $A_{3}^{-}$(beaks) and of $A_{4}$ (butterfly) also generically appear. So we mention here an intrinsic characterization of these three singular points. Let $\left(M^{2}, \mathcal{E},\langle\rangle, D,, \varphi\right)$ be a coherent tangent bundle over a 2-manifold $M^{2}$.

Definition 2.6 (Lips). A $\varphi$-singular point $p \in M^{2}$ is called a lips of $\varphi$ if it satisfies the following conditions: 

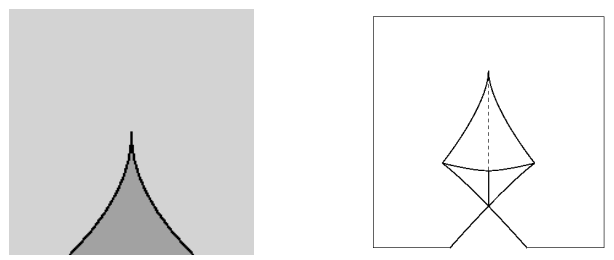

Figure 2. A cusp in a plane (left) and a swallowtail in a space (right).

(1) The rank of $\varphi$ at $p$ is 1 ,

(2) the exterior derivative $d \lambda_{\varphi}$ vanishes at $p$,

(3) the Hessian matrix of the function $\lambda_{\varphi}$ at $p$ is positive definite.

Definition 2.7 (Beaks). A $\varphi$-singular point $p \in M^{2}$ is called a beaks of $\varphi$ if it satisfies the following conditions:

(1) The rank of $\varphi$ at $p$ is 1 ,

(2) the exterior derivative $d \lambda_{\varphi}$ vanishes at $p$,

(3) the Hessian matrix of the function $\lambda_{\varphi}$ at $p$ is negative definite,

(4) the second derivative $\lambda_{\varphi}^{\prime \prime}\left(=d \lambda_{\varphi}^{\prime}(\eta)\right)$ does not vanish at $p$, where the prime means the derivative with respect to the null direction (cf. (2.4) and (2.5)).

Definition 2.8 (Butterfly). A non-degenerate $\varphi$-singular point $p \in M^{2}$ is called a butterfly of $\varphi$ if it satisfies

$$
\lambda_{\varphi}^{\prime}(p)=\lambda_{\varphi}^{\prime \prime}(p)=0, \quad \lambda_{\varphi}^{\prime \prime \prime}(p) \neq 0,
$$

where the prime means the derivative with respect to the null direction.

Remark 2.9. When a coherent tangent bundle is induced from a front, lips, beaks and butterfly correspond to cuspidal lips, cuspidal beaks and cuspidal butterfly, respectively (Fig. 3). See [5] and [4. On the other hand, if a coherent tangent bundle is induced from a smooth map between 2-manifolds, these singular points are corresponding to those on the map (Fig. 4). See [12.

In this way, our intrinsic formulation can give a unified treatment of singular points on maps and on fronts at the same time.

2.4. Singular curvatures. Let $\left(M^{m}, \mathcal{E},\langle\rangle, D,, \varphi\right)$ be a coherent tangent bundle and fix a $\varphi$-singular point $p \in \Sigma_{\varphi}$ which is an $A_{2}$-point. Then there exists a neighborhood $U$ of $p$ such that $\Sigma_{\varphi} \cap U$ consists of $A_{2}$-points. Now we define the singular shape operator as follows: Since the kernel of $\varphi_{p}$ is transversal to $\Sigma_{\varphi}$ at $p,\left.\varphi\right|_{T\left(\Sigma_{\varphi} \cap U\right)}$ is injective, where $U$ is a sufficiently small neighborhood of $p$. Then the metric $d s_{\varphi}^{2}$ is positive definite on $\Sigma_{\varphi} \cap U$. We take an orthonormal frame field $e_{1}, e_{2}, \ldots, e_{m-1}$ on $\Sigma_{\varphi} \cap U$ with respect to $d s_{\varphi}^{2}$. Without loss of generality, we may assume that $\left(e_{1}, e_{2}, \ldots, e_{m-1}\right)$ is smoothly extended on $U$ as an orthonormal $(m-1)$-frame field. Then we can take a unique smooth section $n: U \rightarrow \mathcal{E}$ (called the conormal vector field) so that $\left(\varphi\left(e_{1}\right), \ldots, \varphi\left(e_{m-1}\right), \boldsymbol{n}\right)$ gives a positively oriented orthonormal frame field on $\mathcal{E}$. Now, we set

$$
S_{\varphi}(X):=-\operatorname{sgn}\left(d \lambda_{\varphi}(\eta(q))\right) \varphi^{-1}\left(D_{X} \boldsymbol{n}\right) \quad\left(X \in T_{q} \Sigma_{\varphi}, q \in \Sigma_{\varphi} \cap U\right),
$$



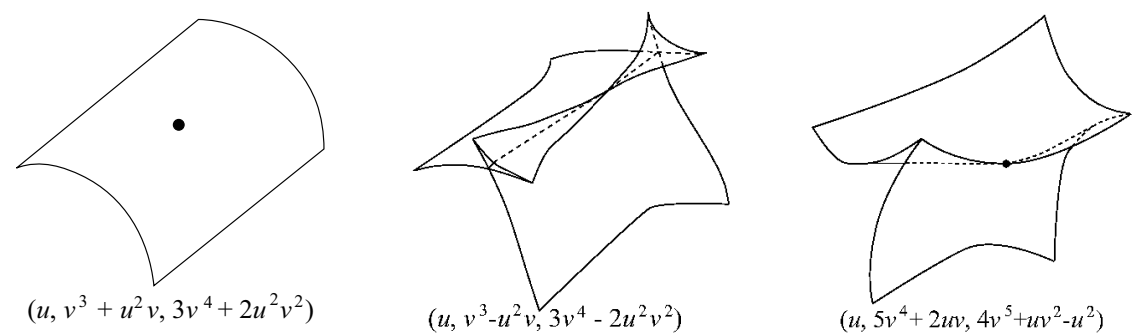

Figure 3. A cuspidal lips (left), a cuspidal beaks (center) and a cuspidal butterfly (right) in $\boldsymbol{R}^{3}$.
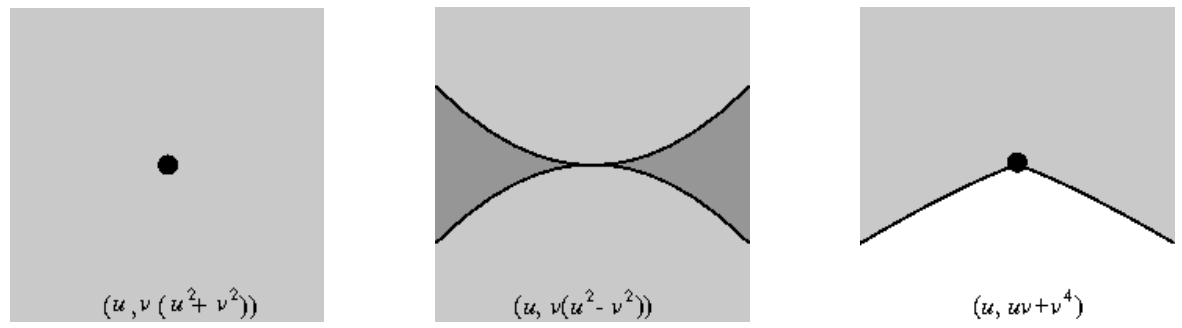

Figure 4. A lips (left), a beaks (center) and a butterfly (right) in $\boldsymbol{R}^{2}$.

where the non-vanishing null vector field $\eta$ is chosen so that $\left(e_{1}, \ldots, e_{m-1}, \eta\right)$ is compatible with respect to the orientation of $M^{m}$. It holds that

$$
\operatorname{sgn}\left(d \lambda_{\varphi}(\eta(q))\right)=\left\{\begin{aligned}
1 & \text { if } \eta(q) \text { points toward } M_{\varphi}^{+}, \\
-1 & \text { if } \eta(q) \text { points toward } M_{\varphi}^{-} .
\end{aligned}\right.
$$

Since $\varphi$ is injective on each tangent space of $\Sigma_{\varphi}$ and $D_{X} \boldsymbol{n} \in \varphi\left(T \Sigma_{\varphi}\right)$, the inverse element $\varphi^{-1}\left(D_{X} \boldsymbol{n}\right)$ is uniquely determined. Thus we get a bundle endomorphism $S_{\varphi}: T \Sigma_{\varphi} \rightarrow T \Sigma_{\varphi}$ which is called the singular shape operator on $\Sigma_{\varphi}$.

Proposition 2.10 (A generalization of Theorem 1.6 in 13]). The definition of the singular shape operator $S_{\varphi}$ is independent of the choice of an orthonormal frame field $e_{1}, \ldots, e_{m-1}$, the choice of an orientation of $M^{m}$, and the choice of a coorientation of $\mathcal{E}$. Moreover, it holds that

$$
d s_{\varphi}^{2}\left(S_{\varphi}(X), Y\right)=d s_{\varphi}^{2}\left(X, S_{\varphi}(Y)\right) \quad\left(X, Y \in T_{q} \Sigma_{\varphi}, q \in \Sigma_{\varphi}\right) .
$$

Proof. In fact, if one reverses the orientation of the basis $\left(e_{1}, \ldots, e_{m-1}\right)$, then $\boldsymbol{n}$ and $\eta$ change sign at the same time, and thus $S_{\varphi}$ is unchanged. Similarly, if we reverse the orientation of $M^{m}$ (resp. $\mathcal{E}$ ), then $\lambda_{\varphi}$ and $\eta$ (resp. $\lambda_{\varphi}$ and $\boldsymbol{n}$ ) change sign at the same time, and $S_{\varphi}$ unchanged.

We can get the final assertion from the following identity

$$
\begin{aligned}
& d s_{\varphi}^{2}\left(\varphi^{-1}\left(D_{X} \boldsymbol{n}\right), Y\right)=\left\langle D_{X} \boldsymbol{n}, \varphi(Y)\right\rangle=-\left\langle\boldsymbol{n}, D_{X} \varphi(Y)\right\rangle \\
& \quad=-\left\langle\boldsymbol{n}, D_{Y} \varphi(X)+\varphi([X, Y])\right\rangle \\
& \quad=-\left\langle\boldsymbol{n}, D_{Y} \varphi(X)\right\rangle=\left\langle D_{Y} \boldsymbol{n}, \varphi(X)\right\rangle=d s_{\varphi}^{2}\left(\varphi^{-1}\left(D_{Y} \boldsymbol{n}\right), X\right),
\end{aligned}
$$

where $X$ and $Y$ are both vector fields on $\Sigma_{\varphi}$. 
Hence $S_{\varphi}$ is symmetric with respect to $d s_{\varphi}^{2}$.

Definition 2.11. Let $p \in \Sigma_{\varphi}$ be an $A_{2}$-point of $\varphi$. Then

$$
\kappa_{\varphi}(X):=d s_{\varphi}^{2}\left(S_{\varphi}(X), X\right) / d s_{\varphi}^{2}(X, X), \quad\left(X \in T_{p} \Sigma_{\varphi} \backslash\{0\}\right)
$$

is called the $\varphi$-singular normal curvature at $p$ with respect to the direction $X$. The eigenvalues of $S_{\varphi}$ are called the $\varphi$-singular principal curvatures, which give the critical values of the singular normal curvature on $T_{p} \Sigma_{\varphi}$.

When $m=2$, the $\varphi$-singular principal curvature is called (simply) the $\varphi$-singular curvature, which is also denoted by $\kappa_{\varphi}$. This definition of the singular curvature is the same as in [13, (1.7)] and [14, (1.6)]. More precisely, $\kappa_{\varphi}$ is computed as follows: Let $p \in \Sigma_{\varphi}$ be an $A_{2}$-point of $\varphi$. Then the $\varphi$-singular set $\Sigma_{\varphi}$ is parametrized by a regular curve $\gamma(t)(t \in I \subset \boldsymbol{R})$ on $M^{2}$ on a neighborhood of $p$, and $\gamma(t)$ is an $A_{2}$-point of $\varphi$ for each $t \in I$. Since $\dot{\gamma}(t)\left({ }^{*}=d / d t\right)$ is not a null-direction, $\varphi(\dot{\gamma}(t)) \neq 0$. Take a section $\boldsymbol{n}(t)$ of $\mathcal{E}$ along $\gamma$ such that $\{\varphi(\dot{\gamma}) /|\varphi(\dot{\gamma})|, \boldsymbol{n}\}$ gives a positive orthonormal frame field on $\mathcal{E}$ along $\gamma$, where $|\varphi(\dot{\gamma})|=\langle\varphi(\dot{\gamma}), \varphi(\dot{\gamma})\rangle^{1 / 2}$. Then we have

$$
\kappa_{\varphi}(t):=\kappa_{\varphi}(\dot{\gamma}(t))=-\operatorname{sgn}\left(d \lambda_{\varphi}(\eta(t))\right) \frac{\left\langle D_{d / d t} \boldsymbol{n}(t), \varphi(\dot{\gamma}(t))\right\rangle}{|\varphi(\dot{\gamma}(t))|^{2}},
$$

where $\eta(t)$ is a null-vector field along $\gamma(t)$ such that $\{\dot{\gamma}(t), \eta(t)\}$ is compatible with the orientation of $M^{2}$. By (2.12), it holds that

$$
\operatorname{sgn}\left(d \lambda_{\varphi}(\eta(t))\right)=\left\{\begin{aligned}
1 & \text { if } M_{\varphi}^{+} \text {lies on the left-hand side of } \gamma \\
-1 & \text { if } M_{\varphi}^{-} \text {lies on the left-hand side of } \gamma
\end{aligned}\right.
$$

2.5. Behavior of the singular curvatures. We now prove the following:

Theorem 2.12 (A generalization of [13, Corollary 1.14]). Let $p \in \Sigma_{\varphi}$ be a $\varphi$ singular point of a coherent tangent bundle $\left(M^{m}, \mathcal{E},\langle\rangle, D,, \varphi\right)$, and assume $p$ is non-degenerate but not an $A_{2}$-point of $\varphi$. Take a regular curve $\gamma:[0,1] \ni t \mapsto \gamma(t) \in$ $\Sigma_{\varphi}$ such that such that $\gamma((0,1])$ consists only of $A_{2}$-points of $\varphi$ and $\gamma(0)=p$. Then one of the $\varphi$-singular principal curvatures along $\gamma(t)$ diverges to $-\infty$.

Proof. We can take a local coordinate system $\left(U ; u_{1}, \ldots, u_{m}\right)$ on $M^{m}$ at $p$ satisfying the following properties:

(1) $\left(u_{1}, \ldots, u_{m}\right)$ is compatible with respect to the orientation of $M^{m}$,

(2) the $\varphi$-singular submanifold is characterized by the zeros of the last coordinate function, that is, $\Sigma_{\varphi} \cap U=\left\{u_{m}=0\right\}$.

(3) the vector field $\eta:=\frac{\partial}{\partial u_{1}}+\delta \frac{\partial}{\partial u_{m}}$ gives the null direction, where $\delta:=$ $\delta\left(u_{1}, \ldots, u_{m-1}\right)$ is a $C^{\infty}$-function satisfying $\delta(p)=0$. (In fact, $\eta(p)$ must tangent to the singular manifold, since $p$ is not an $A_{2}$-point.)

For the sake of simplicity, we set

$$
\partial_{j}:=\frac{\partial}{\partial u_{j}}, \quad \varphi_{j}:=\varphi\left(\partial_{j}\right), \quad D_{j}:=D_{\partial_{j}} \quad(j=1, \ldots, m) .
$$

Since $\gamma(t)(t \in(0,1])$ is an $A_{2}$-point of $\varphi,(2)$ and $(3)$ imply that $\delta(t):=\delta(\gamma(t))$ does not vanish for $t \in(0,1]$. Then $\left\{\partial_{1}, \ldots, \partial_{m-1}, \operatorname{sgn}(\delta) \eta\right\}$ forms a positive frame 
field on $T M^{m}$ along $\gamma(t)(t \neq 0)$. By definition, the $\varphi$-singular normal curvature along $\gamma$ with respect to $\partial_{1}$ is given by

$$
\begin{aligned}
\kappa_{\varphi}(t)(: & \left.=\kappa_{\varphi}\left(\partial_{1}\right)\right)=-\operatorname{sgn}\left(d \lambda_{\varphi}(\operatorname{sgn}(\delta) \eta)\right) \frac{\left\langle D_{1} \boldsymbol{n}, \varphi_{1}\right\rangle}{\left|\varphi_{1}\right|^{2}} \\
& =-\operatorname{sgn}(\delta) \operatorname{sgn}\left(d \lambda_{\varphi}(\eta)\right) \frac{\left\langle\boldsymbol{n}, D_{1} \varphi_{1}\right\rangle}{\left|\varphi_{1}\right|^{2}} .
\end{aligned}
$$

Since $\eta$ is a null vector, it holds that

$$
\varphi(\eta)=\varphi_{1}+\delta \varphi_{m}=0
$$

on $\Sigma_{\varphi}$. In particular, since $\eta=\partial_{1}$ at $p$, we have that

$$
\varphi_{m}(p) \neq 0 \text {. }
$$

Differentiating (2.18) by $u_{1}$, we have

$$
D_{1} \varphi_{1}+\delta_{1} \varphi_{m}+\delta D_{1} \varphi_{m}=0 \quad\left(\delta_{1}:=\frac{\partial \delta}{\partial u_{1}}\right) .
$$

We can identify $\bigwedge_{j=1}^{m-1} \mathcal{E}_{q} \cong \mathcal{E}_{q}$ for each $q \in M^{m}$ by using the inner product on $\mathcal{E}$, and then we set

$$
\boldsymbol{n}=\frac{\varphi_{1} \wedge \cdots \wedge \varphi_{m-1}}{\left|\varphi_{1} \wedge \cdots \wedge \varphi_{m-1}\right|},
$$

which gives a conormal vector field such that $\left\{\varphi_{1}, \ldots, \varphi_{m-1}, \boldsymbol{n}\right\}$ is a positive frame field on $\mathcal{E}$ along $\gamma(t)(t \neq 0)$.

By (2.18), we have $\varphi_{1}=-\delta \varphi_{m}$. Then

$$
\boldsymbol{n}=-\frac{\delta}{|\delta|} \cdot \frac{\varphi_{m} \wedge \varphi_{2} \wedge \cdots \wedge \varphi_{m-1}}{\left|\varphi_{m} \wedge \varphi_{2} \wedge \cdots \wedge \varphi_{m-1}\right|}=(-1)^{m-1} \operatorname{sgn}(\delta) \frac{\varphi_{2} \wedge \cdots \wedge \varphi_{m}}{\left|\varphi_{2} \wedge \cdots \wedge \varphi_{m}\right|} .
$$

Substituting (2.18) and (2.20) into (2.17), we have that

$$
\begin{aligned}
\kappa_{\varphi}(t) & =-\operatorname{sgn}(\delta) \operatorname{sgn}\left(d \lambda_{\varphi}(\eta)\right) \frac{\left\langle\boldsymbol{n}, \delta_{1} \varphi_{m}+\delta D_{1} \varphi_{m}\right\rangle}{\left|\delta \varphi_{m}\right|^{2}} \\
& =-\operatorname{sgn}\left(d \lambda_{\varphi}(\eta)\right) \frac{\left\langle\boldsymbol{n}, D_{1} \varphi_{m}\right\rangle}{|\delta|\left|\varphi_{m}\right|^{2}} .
\end{aligned}
$$

Set

$$
\Delta:=\frac{(-1)^{m-1} \operatorname{sgn}\left(d \lambda_{\varphi}(\eta)\right)}{|\delta|\left|\varphi_{m}\right|^{2}\left|\varphi_{2} \wedge \cdots \wedge \varphi_{m}\right|} \quad \text { and } \quad \lambda_{m}:=\frac{\partial \lambda_{\varphi}}{\partial u_{m}} .
$$

Then substituting (2.22) into the above equation and noticing that $\varphi_{1}$ is proportional to $\varphi_{m}$, we have

$$
\begin{aligned}
\frac{\kappa_{\varphi}(t)}{\Delta} & =\left\langle\varphi_{2} \wedge \cdots \wedge \varphi_{m}, D_{m} \varphi_{1}\right\rangle \\
& =\partial_{m}\left\langle\varphi_{2} \wedge \cdots \wedge \varphi_{m}, \varphi_{1}\right\rangle-\left\langle D_{m}\left(\varphi_{2} \wedge \cdots \wedge \varphi_{m}\right), \varphi_{1}\right\rangle \\
& =(-1)^{m-1} \partial_{m} \mu\left(\varphi_{1}, \ldots, \varphi_{m}\right)-\left\langle\varphi_{2} \wedge \cdots \wedge D_{m} \varphi_{m}, \varphi_{1}\right\rangle \\
& =(-1)^{m-1} \lambda_{m}+\left\langle\varphi_{2} \wedge \cdots \wedge D_{m}\left(\delta \varphi_{1}\right), \varphi_{1}\right\rangle \\
& =(-1)^{m-1} \lambda_{m}+\delta\left\langle\varphi_{2} \wedge \cdots \wedge D_{m} \varphi_{1}, \varphi_{1}\right\rangle .
\end{aligned}
$$

Hence

$$
\kappa_{\varphi}(t)=\frac{\operatorname{sgn}\left(d \lambda_{\varphi}(\eta)\right) \operatorname{sgn}(\delta)}{|\delta|\left|\varphi_{m}\right|^{2}\left|\varphi_{2} \wedge \cdots \wedge \varphi_{m}\right|} \lambda_{m}+(\text { a bounded term })
$$


because of (2.19). Since $d \lambda_{\varphi}(\eta)=d \lambda_{\varphi}\left(\partial_{1}+\delta \partial_{m}\right)=\delta \lambda_{m}$, we have that

$$
\kappa_{\varphi}(t)=-\frac{\left|\lambda_{m}\right|}{|\delta|\left|\varphi_{m}\right|^{2}\left|\varphi_{2} \wedge \cdots \wedge \varphi_{m}\right|}+(\text { a bounded term }) .
$$

Since $p$ is non-degenerate and $\Sigma_{\varphi}=\left\{u_{m}=0\right\}, \lambda_{m} \neq 0$ holds. Thus, $\kappa_{\varphi}(t)$ tends to $-\infty$ as $t \rightarrow+0$. Hence, at least one of the singular principal curvatures tends to $-\infty$

2.6. Frontal bundles. At the end of this section, we give a definition of frontal bundles as an intrinsic characterization of wave fronts in space forms.

Let $M^{m}$ be an oriented $m$-manifold and $\left(M^{m}, \mathcal{E},\langle\rangle, D,, \varphi\right)$ a co-orientable coherent tangent bundle over $M^{m}$. If there exists another bundle homomorphism $\psi: T M^{m} \rightarrow \mathcal{E}$ such that $\left(M^{m}, \mathcal{E},\langle\rangle, D,, \psi\right)$ is also a coherent tangent bundle and the pair $(\varphi, \psi)$ of bundle homomorphisms satisfies a compatibility condition

$$
\langle\varphi(X), \psi(Y)\rangle=\langle\varphi(Y), \psi(X)\rangle,
$$

then $\left(M^{m}, \mathcal{E},\langle\rangle, D,, \varphi, \psi\right)$ is called a frontal bundle. The bundle homomorphisms $\varphi$ and $\psi$ are called the first homomorphism and the second homomorphism, respectively. We set

$$
\begin{aligned}
I(X, Y) & :=d s_{\varphi}^{2}(X, Y)=\langle\varphi(X), \varphi(Y)\rangle, \\
I I(X, Y) & :=-\langle\varphi(X), \psi(Y)\rangle, \\
I I I(X, Y) & :=d s_{\psi}^{2}(X, Y)=\langle\psi(X), \psi(Y)\rangle
\end{aligned}
$$

for $X, Y \in T_{p} M^{m}\left(p \in M^{m}\right)$, and we call them the first, the second and the third fundamental forms, respectively. They are all symmetric covariant tensors on $M^{m}$.

Definition 2.13. A frontal bundle $\left(M^{m}, \mathcal{E},\langle\rangle, D,, \varphi, \psi\right)$ is called a front bundle if

$$
\operatorname{Ker}\left(\varphi_{p}\right) \cap \operatorname{Ker}\left(\psi_{p}\right)=\{0\}
$$

holds for each $p \in M^{m}$.

Example 2.14. Let $\left(N^{m+1}(c), g\right)$ be an $(m+1)$-dimensional space form, that is, a complete Riemannian $(m+1)$-manifold of constant curvature $c$, and denote by $\nabla$ the Levi-Civita connection on $N^{m+1}(c)$. Let $f: M^{m} \rightarrow N^{m+1}(c)$ be a co-orientable frontal. Then there exists a globally defined unit normal vector field $\nu$. Since the coherent tangent bundle $\mathcal{E}_{f}$ given in Example 2.4 is orthogonal to $\nu$, we can define a bundle homomorphism

$$
\psi_{f}: T_{p} M^{m} \ni X \longmapsto \nabla_{X} \nu \in \mathcal{E}_{p} \quad\left(p \in M^{m}\right) .
$$

Then $\left(M^{m}, \mathcal{E}_{f},\langle\rangle, D,, \varphi_{f}, \psi_{f}\right)$ is a frontal bundle. Moreover, this is a front bundle in the sense of Definition 2.13 if and only if $f$ is a front, which is equivalent to $I+I I I$ being positive definite. As a fundamental theorem for hypersurfaces, the integrability condition for a given frontal bundle to be realized as a frontal in a space form is given in 18 .

We fix a front bundle $\left(M^{m}, \mathcal{E},\langle\rangle, D,, \varphi, \psi\right)$ over an $m$-dimensional manifold $M^{m}$. 
Definition 2.15. When $p \in M^{m}$ is not a singular point of $\varphi$, we define

$$
K^{\mathrm{ext}}(X \wedge Y):=\frac{I I(X, X) I I(Y, Y)-I I(X, Y)^{2}}{I(X, X) I(Y, Y)-I(X, Y)^{2}} \quad\left(X, Y \in T_{p} M^{m}\right),
$$

which is called the extrinsic curvature at $p$ with respect to the $X \wedge Y$-plane in $T_{p} M^{m}$.

If a front bundle $\left(M^{m}, \mathcal{E},\langle\rangle, D,, \varphi, \psi\right)$ is induced from a front in $N^{m+1}(c)$, then it holds that

$$
K^{\operatorname{ext}}(X \wedge Y)=K(X \wedge Y)+c \quad\left(X, Y \in T_{p} M^{m}\right),
$$

where $K(X \wedge Y)$ is the sectional curvature at each $\varphi$-regular point $p$ of $M^{m}$.

As a generalization of [13, Theorem 3.1], relationships between singular principal curvatures and $K^{\text {ext }}$ of wave fronts in space forms are investigated in [18.

\section{Four Gauss-Bonnet formulas on Surfaces}

In this section, we give four Gauss-Bonnet formulas on a given front in 3dimensional space forms, and will point out several remarkable consequences of them.

3.1. The Gauss-Bonnet formulas for smooth maps. Let $M^{m}$ be an oriented $m$-manifold and $\left(N^{m}, g\right)$ an oriented Riemannian $m$-manifold. As in Example 2.3, a $C^{\infty}$-map $f: M^{m} \rightarrow N^{m}$ induces a coherent tangent bundle $\left(M^{m}, \mathcal{E}_{f},\langle\rangle, D,, \varphi_{f}=\right.$ $d f$ ) over $M^{m}$. In this setting, an $A_{k}$-point (cf. Definitions 2.1 and 2.2) coincides with an $A_{k}$-Morin singular point of $f$ (see [15]).

Now we restrict our attention to the case $m=2$ : An $A_{2}$-point (resp. $A_{3}$-point) of $\varphi_{f}$ on $M^{2}$ is called a fold (resp. a cusp); namely, a fold (resp. a cusp) is right-left equivalent to the map

$$
\boldsymbol{R}^{2} \ni(u, v) \longmapsto\left(u^{2}, v\right) \in \boldsymbol{R}^{2} \quad\left(\operatorname{resp} . \boldsymbol{R}^{2} \ni(u, v) \longmapsto\left(u v+v^{3}, u\right) \in \boldsymbol{R}^{2}\right)
$$

at the origin. Here, two map germs $f_{i}:\left(\boldsymbol{R}^{m}, p\right) \rightarrow\left(\boldsymbol{R}^{n}, q\right)(i=1,2)$ are rightleft equivalent if there exist diffeomorphism germs $\xi_{1}:\left(\boldsymbol{R}^{m}, p\right) \rightarrow\left(\boldsymbol{R}^{m}, p\right)$ and $\xi_{2}:\left(\boldsymbol{R}^{n}, q\right) \rightarrow\left(\boldsymbol{R}^{n}, q\right)$ such that $\xi_{2} \circ f_{1}=f_{2} \circ \xi_{1}$ holds.

The $\varphi_{f}$-singular curvature $\kappa_{f}(p)$ of an $A_{2}$-point $p$ of $\varphi_{f}$ (i.e. a fold) is called the singular curvature at a fold. The following assertion follows immediately.

Proposition 3.1. Let $f: M^{2} \rightarrow N^{2}$ be a $C^{\infty}{ }_{-}$map and $p$ a fold singular point. Suppose that $\gamma(t)$ is a regular curve which parametrizes the singular set so that $f\left(M^{2}\right)$ lies on the left-hand side of $f \circ \gamma$. Then the singular curvature $\kappa_{f}(p)$ at $p$ is equal to the geodesic curvature of $f \circ \gamma$.

Example 3.2. We set $f_{\epsilon}(u, v):=\left(u^{2}+\epsilon v^{2}, v\right)(\epsilon:= \pm 1)$. If $\epsilon=1$ (resp. $\left.\epsilon=-1\right)$, then all singular points consist of folds with positive (resp. negative) singular curvature, see Fig. 5

Now we recall the following two Gauss-Bonnet formulas on a given coherent tangent bundle:

Fact 3.3 (13, 14). Let $\left(M^{2}, \mathcal{E},\langle\rangle, D,, \varphi\right)$ be a coherent tangent bundle over a compact oriented 2-manifold $M^{2}$, and suppose that the $\varphi$-singular set $\Sigma_{\varphi}$ consists 

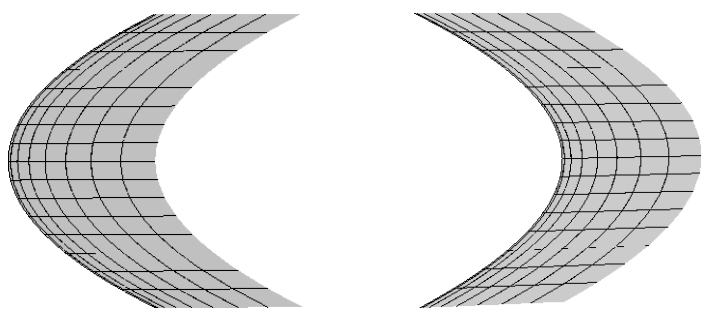

Figure 5. The images of $f_{1}$ and $f_{-1}$ (the folds are the left edges in both figures).

of $A_{2}$-points and $A_{3}$-points. We denote by $K$ the Gaussian curvature of the induced metric $d s^{2}=\varphi^{*}\langle$,$\rangle . Then it holds that$

$$
\begin{aligned}
& \left(\chi_{\mathcal{E}}=\right) \frac{1}{2 \pi} \int_{M^{2}} K d \hat{A}_{\varphi}=\chi\left(M_{\varphi}^{+}\right)-\chi\left(M_{\varphi}^{-}\right)+S_{\varphi}^{+}-S_{\varphi}^{-}, \\
& 2 \pi \chi\left(M^{2}\right)=\int_{M^{2}} K d A_{\varphi}+2 \int_{\Sigma_{\varphi}} \kappa_{\varphi} d \tau_{\varphi},
\end{aligned}
$$

where $d \tau_{\varphi}$ is the length element on the $\varphi$-singular set with respect to $d s_{\varphi}^{2}$, and $S_{\varphi}^{+}$ and $S_{\varphi}^{-}$are the numbers of positive and negative $A_{3}$-points of $\varphi$, respectively (see [13. Figure 4] and also [14, Definition 2.9]). On the other hand, $\chi_{\mathcal{E}}$ is the Euler characteristic of the $\mathrm{SO}(2)$-vector bundle $\mathcal{E}$.

Remark 3.4. Let $(\mathcal{E},\langle\rangle$,$) be an oriented vector bundle of rank 2$ with a metric over a compact oriented 2-manifold $M^{2}$, and $D$ a metric connection. Suppose that there exists a bundle homomorphism $\varphi: T M^{2} \rightarrow \mathcal{E}$. In the first section, $A_{2}$ and $A_{3}$-points are defined for such an arbitrary bundle homomorphism $\varphi$ and (3.1) holds without assuming the compatibility condition (2.6), as pointed out in 17: For an oriented orthonormal frame field $\left\{\boldsymbol{e}_{1}, \boldsymbol{e}_{2}\right\}$ of $\mathcal{E}$ defined on $U \subset M^{2}$, there is a unique 1-form $\omega$ on $U$ such that

$$
D_{X} \boldsymbol{e}_{1}=-\omega(X) \boldsymbol{e}_{2}, \quad D_{X} \boldsymbol{e}_{2}=\omega(X) \boldsymbol{e}_{1} .
$$

Then $d \omega$ does not depend on the choice of $\left\{\boldsymbol{e}_{1}, \boldsymbol{e}_{2}\right\}$, and there is a $C^{\infty}$-function $K_{\varphi, D}$ on $M^{2} \backslash \Sigma_{\varphi}$ such that

$$
d \omega=K_{\varphi, D} d \hat{A}_{\varphi}
$$

We call $K_{\varphi, D}$ the Gaussian curvature of $D$ with respect to $\varphi$. If $\mathcal{E}$ is a coherent tangent bundle, the identity (2.6) implies that $K_{\varphi, D}$ coincides with the Gaussian curvature of the metric $d s_{\varphi}^{2}=\varphi^{*}\langle$,$\rangle . Thus formulas (3.1) and (3.2) still hold$ for $K=K_{\varphi, D}$, without assuming (2.6). Although (3.2) depends on $D$, (3.1) is independent of the choice of metric connections.

Remark 3.5. Under the assumption that $\mathcal{E}$ is a coherent tangent bundle, the identities (3.1) and (3.2) hold even if $\varphi$ admits wider class of singular points called 'peaks'. (cf. [13, Definition 1.10] and also [14, Definition 2.1]). $A_{3}$-points, lips (see Definition 2.6), beaks (see Definition 2.7), butterflies (see Definition 2.8) are all examples of peaks. For a coherent tangent bundle over a compact oriented 2-manifold whose singular points are at most peaks, the formulas (3.1) and (3.2) hold (see 13. 
Theorem 2.3] and [14, Theorem B]), here $S_{\varphi}^{+}$and $S_{\varphi}^{-}$in (3.1) should be replaced by the numbers of positive (resp. negative) peaks (cf. [14, Definition 2.1]).

The formula (3.1) in our situation induces Quine's formula.

Proposition 3.6 (Quine [10). Let $M^{2}$ and $N^{2}$ both be compact oriented connected 2-manifolds, and $f: M^{2} \rightarrow N^{2}$ be a $C^{\infty}$-map whose singular set consists of folds and cusps. Then the topological degree of $f$ satisfies

$$
\operatorname{deg}(f) \chi\left(N^{2}\right)=\chi\left(M_{f}^{+}\right)-\chi\left(M_{f}^{-}\right)+S_{f}^{+}-S_{f}^{-},
$$

where $M_{f}^{+}$(resp. $\left.M_{f}^{-}\right)$is the set of regular points at which $f$ preserves (resp. reverses) the orientation, and $S_{f}^{+}$(resp. $S_{f}^{-}$) is the number of positive cusps (resp. the number of negative cusps).

On the other hand, the formula (3.2) induces the following new formula.

Proposition 3.7. Let $\left(N^{2}, g\right)$ be an oriented Riemannian 2-manifold, and $M^{2}$ a compact oriented 2-manifold. Let $f: M^{2} \rightarrow N^{2}$ be a $C^{\infty}$-map whose singular set consists of folds and cusps. Then the total singular curvature $\int_{\Sigma} \kappa d \tau$ with respect to the length element $d \tau$ (with respect to $g$ ) on the singular set $\Sigma$ is bounded, and satisfies the following identity

$$
2 \pi \chi\left(M^{2}\right)=\int_{M^{2}}(\widetilde{K} \circ f)\left|f^{*} d A_{g}\right|+2 \int_{\Sigma} \kappa d \tau
$$

where $\widetilde{K}$ is the Gaussian curvature function on $\left(N^{2}, g\right)$, and $\left|f^{*} d A_{g}\right|$ is the pull-back of the Riemannian measure of $\left(N^{2}, g\right)$.

In particular, if $\left(N^{2}, g\right)$ is the Euclidean plane $\left(\boldsymbol{R}^{2}, g_{0}\right)$, we have the following classical result:

Corollary 3.8 (Levine [8). Let $M^{2}$ be a compact oriented 2-manifold, and $f$ : $M^{2} \rightarrow \boldsymbol{R}^{2}$ a $C^{\infty}$-map whose singular set consists of folds and cusps. Let $C_{1}, \ldots, C_{r}$ be the disjoint union of the closed regular curves on $M^{2}$ such that the singular set of $f$ is $C_{1} \cup \cdots \cup C_{r}$. Suppose that we give an orientation to each $f\left(C_{j}\right)(j=1, \ldots, r)$ so that the image $f\left(M^{2}\right)$ lies on the left-hand side of $f\left(C_{j}\right)$. Then the rotation indices $I\left(C_{j}\right)$ (which takes values in the set of half-integers) of $f\left(C_{j}\right)(j=1, \ldots, r)$ as fronts satisfy

$$
\frac{\chi\left(M^{2}\right)}{2}=I\left(C_{1}\right)+\ldots+I\left(C_{r}\right)
$$

3.2. The Gauss-Bonnet formulas for wave fronts. We use the same notation as in the previous sections. Let $N^{3}(c)$ be a 3-dimensional complete Riemannian manifold with constant curvature $c$. Here, we do not assume that $N^{3}(c)$ is simply connected, and we use the notation $\widetilde{N}^{3}(c)$ when it is in fact assumed to be simply connected, like in the previous section.

Let $M^{2}$ be an oriented closed 2-manifold and $f: M^{2} \rightarrow N^{3}(c)$ a front. Then the front bundle $\left(M^{2}, \mathcal{E}_{f},\langle\rangle, D,, \varphi=\varphi_{f}, \psi=\psi_{f}\right)$ is defined as in Example 2.14 We fix a positively oriented local coordinate system $(U ; u, v)$ on $M^{2}$. Denote by $\lambda$ (resp. $\lambda_{\#}$ ) the $\varphi$-Jacobian function (resp. $\psi$-Jacobian function) as in (2.1):

$$
\lambda:=\lambda_{\varphi}=\mu\left(\varphi_{u}, \varphi_{v}\right) \quad \text { and } \quad \lambda_{\#}:=\lambda_{\psi}=\mu\left(\psi_{u}, \psi_{v}\right),
$$

where

$$
\varphi_{u}=\varphi(\partial / \partial u), \quad \varphi_{v}=\varphi(\partial / \partial v), \quad \psi_{u}=\psi(\partial / \partial u), \quad \psi_{v}=\psi(\partial / \partial v),
$$


and $\mu$ is the volume form of $\mathcal{E}_{f}$ giving co-orientation of $\mathcal{E}_{f}$. Then the $\varphi$-singular set (resp. $\psi$-singular set)

$$
\Sigma:=\Sigma_{\varphi} \quad\left(\operatorname{resp} . \Sigma_{\#}:=\Sigma_{\psi}\right)
$$

is expressed as

$$
\Sigma \cap U=\{p \in U ; \lambda(p)=0\} \quad\left(\text { resp. } \Sigma_{\#} \cap U=\left\{p \in U ; \lambda_{\#}(p)=0\right\}\right) .
$$

Remark 3.9. If $N^{3}(c)=\boldsymbol{R}^{3}, \Sigma_{\#}$ is the singular set of the Gauss map $\nu: M^{2} \rightarrow S^{2}$ of $f$. When $N^{3}(c)=S^{3}, \Sigma_{\#}$ is the singular set of the map induced by the unit normal vector field $\nu: M^{2} \rightarrow S^{3}$, where $S^{3}$ is the unit 3-sphere. This unit normal vector corresponds to the dual map of $f$ induced by a duality between $S^{3}$ and itself. When $N^{3}(c)$ is the hyperbolic 3-space $H^{3}, \Sigma_{\#}$ is the singular set of the map induced by the normal vector field $\nu: M^{2} \rightarrow S_{1}^{3}$ into the de Sitter 3 -space $S_{1}^{3}$.

We write the volume forms as in (2.10) as

$$
\begin{aligned}
& d \hat{A}:=d \hat{A}_{\varphi}=\lambda d u \wedge d v, \quad d \hat{A}_{\#} \quad:=d \hat{A}_{\psi}=\lambda_{\#} d u \wedge d v, \\
& d A:=d A_{\varphi}=|\lambda| d u \wedge d v, \quad d A_{\#} \quad:=d A_{\psi}=\left|\lambda_{\#}\right| d u \wedge d v,
\end{aligned}
$$

and

$$
\begin{aligned}
& M^{+}:=M_{\varphi}^{+}=\left\{p \in M^{2} \backslash \Sigma ; d \hat{A}=d A\right\}, \\
& M^{-}:=M_{\varphi}^{-}=\left\{p \in M^{2} \backslash \Sigma ; d \hat{A}=-d A\right\}, \\
& M_{\#}^{+}:=M_{\psi}^{+}=\left\{p \in M^{2} \backslash \Sigma_{\#} ; d \hat{A}_{\#}=d A_{\#}\right\}, \\
& M_{\#}^{-}:=M_{\psi}^{-}=\left\{p \in M^{2} \backslash \Sigma_{\#} ; d \hat{A}_{\#}=-d A_{\#}\right\} .
\end{aligned}
$$

Denote by $K\left(\right.$ resp. $K_{\#}$ ) the Gaussian curvature of the metric $I=d s_{\varphi}^{2}$ (resp. $I I I=d s_{\psi}^{2}$ ), which is defined on $M^{2} \backslash \Sigma\left(\right.$ resp. $\left.M^{2} \backslash \Sigma_{\#}\right)$.

The extrinsic curvature $K^{\text {ext }}$ in Definition 2.15 can be considered as a $C^{\infty}$. function on $M^{2} \backslash \Sigma$, because we are working with the 2-dimensional case. Similarly, we denote by $K_{\#}^{\text {ext }}$ the extrinsic curvature with respect to the second homomorphism $\psi$, namely, it is obtained by replacing $I$ with $I I I$ in 2.25). If we denote by $\widehat{I}, \widehat{I I}$ and $\widehat{I I I}$ the representation matrices of $I, I I$ and $I I I$, respectively, with respect to $\{\partial / \partial u, \partial / \partial v\}:$

$$
\begin{aligned}
\widehat{I} & :=\left(\begin{array}{cc}
\left\langle\varphi_{u}, \varphi_{u}\right\rangle & \left\langle\varphi_{u}, \varphi_{v}\right\rangle \\
\left\langle\varphi_{v}, \varphi_{u}\right\rangle & \left\langle\varphi_{v}, \varphi_{v}\right\rangle
\end{array}\right), \\
\widehat{I I} & :=-\left(\begin{array}{ll}
\left\langle\varphi_{u}, \psi_{u}\right\rangle & \left\langle\varphi_{u}, \psi_{v}\right\rangle \\
\left\langle\varphi_{v}, \psi_{u}\right\rangle & \left\langle\varphi_{v}, \psi_{v}\right\rangle
\end{array}\right), \\
\widehat{I I I} & :=\left(\begin{array}{ll}
\left\langle\psi_{u}, \psi_{u}\right\rangle & \left\langle\psi_{u}, \psi_{v}\right\rangle \\
\left\langle\psi_{v}, \psi_{u}\right\rangle & \left\langle\psi_{v}, \psi_{v}\right\rangle
\end{array}\right),
\end{aligned}
$$

then the extrinsic curvatures are expressed as

$$
K^{\mathrm{ext}}=\frac{\operatorname{det} \widehat{I I}}{\operatorname{det} \widehat{I}}, \quad K_{\#}^{\mathrm{ext}}=\frac{\operatorname{det} \widehat{I I}}{\operatorname{det} \widehat{I I I}} .
$$

Then (2.26) is equivalent to

$$
K=c+K^{\mathrm{ext}} .
$$

Lemma 3.10. Let $f: M^{2} \rightarrow N^{3}(c)$ be a front. Then

(a) $K d \hat{A}=K_{\#} d \hat{A}_{\#}$, 
(b) $K_{\#}=1$ if $c=0$,

(c) $K^{\text {ext }} d \hat{A}=d \hat{A}_{\#}$ and $K_{\#}^{\operatorname{ext}} d \hat{A}_{\#}=d \hat{A}$,

(d) $\left|K^{\mathrm{ext}}\right| d A=d A_{\#}$ and $\left|K_{\#}^{\mathrm{ext}}\right| d A_{\#}=d A$,

(e) $K^{\text {ext }} K_{\#}^{\text {ext }}=1$.

Proof. Take a positive (local) orthonormal frame field $\left\{\boldsymbol{e}_{1}, \boldsymbol{e}_{2}\right\}$ of $\mathcal{E}_{f}$, and take a one-form $\omega$ as in (3.3), that is, $\omega$ is the connection form of $D$ with respect to the frame $\left\{\boldsymbol{e}_{1}, \boldsymbol{e}_{2}\right\}$. By (3.4) $K d \hat{A}$ is determined by just the connection $D$. Hence we have (a) Take $2 \times 2$-matrix-valued functions $G$ and $G_{\#}$ as

$$
\left(\varphi_{u}, \varphi_{v}\right)=G\left(\boldsymbol{e}_{1}, \boldsymbol{e}_{2}\right), \quad\left(\psi_{u}, \psi_{v}\right)=G_{\#}\left(\boldsymbol{e}_{1}, \boldsymbol{e}_{2}\right) .
$$

By definition, $d \hat{A}$ and $d \hat{A}_{\#}$ in (3.6) are expressed as

$$
\begin{gathered}
d \hat{A}=\lambda d u \wedge d v=(\operatorname{det} G) d u \wedge d v, \\
d \hat{A}_{\#}=\lambda_{\#} d u \wedge d v=\left(\operatorname{det} G_{\#}\right) d u \wedge d v .
\end{gathered}
$$

In particular, we have that

$$
d A=|\operatorname{det} G| d u \wedge d v, \quad d A_{\#}=\left|\operatorname{det} G_{\#}\right| d u \wedge d v .
$$

On the other hand, the matrices $\widehat{I}, \widehat{I I}$ and $\widehat{I I I}$ as in (3.7) are written as

$$
\widehat{I}={ }^{t} G G, \quad \widehat{I I}=-{ }^{t} G G_{\#}=-{ }^{t} G_{\#} G, \quad \widehat{I I I}={ }^{t} G_{\#} G_{\#},
$$

where ${ }^{t}()$ denotes transposition. Thus, by (3.8), we have

$$
K^{\mathrm{ext}}=\frac{\operatorname{det} G_{\#}}{\operatorname{det} G}, \quad K_{\#}^{\mathrm{ext}}=\frac{\operatorname{det} G}{\operatorname{det} G_{\#}} .
$$

Hence (c), (d) and (e) hold. Finally, if $c=0, I I I$ is just the pull-back metric of canonical metric of the unit sphere by the Gauss map, so (b) follows.

Now, we assume both of the singular sets $\Sigma$ and $\Sigma_{\#}$ consist of $A_{2^{-}}$points and $A_{3}$-points. Then applying two abstract formulas (3.1) and (3.2) for $\varphi$ and $\psi$ respectively, we have the following four Gauss-Bonnet formulas:

$$
\begin{aligned}
\int_{M^{2}} K d \hat{A} & =\int_{M^{+}} K d A-\int_{M^{-}} K d A \\
& =2 \pi\left(\chi\left(M^{+}\right)-\chi\left(M^{-}\right)\right)+2 \pi\left(S^{+}-S^{-}\right), \\
\int_{M^{2}} K d A & =2 \pi \chi(M)-2 \int_{\Sigma} \kappa d \tau, \\
\int_{M^{2}} K_{\#} d \hat{A}_{\#} & =\int_{M_{\#}^{+}} K_{\#} d A_{\#}-\int_{M_{\#}^{-}} K_{\#} d A_{\#} \\
& =2 \pi\left(\chi\left(M_{\#}^{+}\right)-\chi\left(M_{\#}^{-}\right)\right)+2 \pi\left(S_{\#}^{+}-S_{\#}^{-}\right), \\
\int_{M^{2}} K_{\#} d A_{\#} & =2 \pi \chi\left(M^{2}\right)-2 \int_{\Sigma_{\#}} \kappa_{\#} d \tau_{\#},
\end{aligned}
$$

where $\kappa$ (resp. $\kappa_{\#}$ ) is the singular curvature function along $A_{2}$-points in $\Sigma$ (resp. $\left.\Sigma_{\#}\right)$ as in (2.14),$d \tau$ (resp. $d \tau_{\#}$ ) is the length element on the singular curve with respect to $I$ (resp. III), $S^{+}$(resp. $S^{-}$) is the number of positive (resp. negative) $A_{3^{-}}$ points of $\varphi$, and $S_{\#}^{+}$(resp. $\left.S_{\#}^{-}\right)$is the number of positive (resp. negative) $A_{3}$-points of $\psi$. 
Remark 3.11. As seen in Remark 3.5, formulas (3.14)-3.17) hold for fronts such that the singular sets of $\varphi$ and $\psi$ consist of at most peak. Here, $S^{+}$(resp. $S^{-}$) in (3.14) should be considered as a number of positive (resp. negative) peaks of $\varphi$, and $S_{\#}^{+}\left(\right.$resp. $\left.S_{\#}^{-}\right)$in (3.16) should be considered as a number of positive (resp. negative) peaks of $\psi$, in this case.

3.3. Applications of the four Gauss-Bonnet formulas. Let $f: M^{2} \rightarrow N^{3}(c)$ be an immersion of a compact orientable 2-manifold $M^{2}$. Then the second homomorphism $\psi_{f}$ is the shape operator of $f$, and the set of singular points $\Sigma_{\#}$ of it coincides with the inflection points of $f$ (see [16]). Then $A_{2}$-points and $A_{3}$-points in $\Sigma_{\#}$ are called $A_{2}$-inflection points and $A_{3}$-inflection points, respectively.

Theorem 3.12 (A generalization of the Bleecker-Wilson formula). Let $M^{2}$ be a compact oriented 2-manifold and $f: M^{2} \rightarrow N^{3}(c)$ an immersion. Suppose that the set of inflection points of $f$ consists of $A_{2}$-points and $A_{3}$-points. Then we have

$$
2 \chi\left(M_{\#}^{-}\right)=S_{\#}^{+}-S_{\#}^{-} \text {. }
$$

Moreover, $M_{\#}^{-}$coincides with the set $\left\{p \in M^{2} ; K^{\operatorname{ext}}(p)<0\right\}$.

Proof. Since $A_{2}$-inflection points and $A_{3}$-inflection points are non-degenerate singular points of $\psi$, the set of inflection points $\Sigma_{\#}$ is a regular submanifold of a compact manifold $M^{2}$. Hence $\Sigma_{\#}$ is the disjoint union of a finite number of closed curves in $M^{2}$, and thus the Euler number $\chi\left(\Sigma_{\#}\right)$ vanishes. Then we have

$$
\chi\left(M^{2}\right)=\chi\left(M_{\#}^{+}\right)+\chi\left(M_{\#}^{-}\right)+\chi\left(\Sigma_{\#}\right)=\chi\left(M_{\#}^{+}\right)+\chi\left(M_{\#}^{-}\right) .
$$

Since $f$ is an immersion, $d A=d \hat{A}$ holds, and then

$$
\begin{aligned}
\chi\left(M^{2}\right) & =\frac{1}{2 \pi} \int_{M^{2}} K d \hat{A} & & (\text { by the Gauss-Bonnet formula) } \\
& =\frac{1}{2 \pi} \int_{M^{2}} K_{\#} d \hat{A}_{\#} & & (\text { by (a) in Lemma 3.10) } \\
& =\chi\left(M_{\#}^{+}\right)-\chi\left(M_{\#}^{-}\right)+S_{\#}^{+}-S_{\#}^{-} & & (\text {by (3.16) }) \\
& =\chi\left(M^{2}\right)-2 \chi\left(M_{\#}^{-}\right)+S_{\#}^{+}-S_{\#}^{-} & & (\text {by (3.19) }) .
\end{aligned}
$$

Thus, we have the equality. Here, since $d A=d \hat{A}$, (c) and (d) of Lemma 3.10implies that $\Sigma_{\#}=\left\{p \in M^{2} ; K^{\text {ext }}=0\right\}$. Then

$$
\begin{array}{rlrl}
M_{\#}^{-} & =\left\{p \in M^{2} \backslash \Sigma_{\#} ; d \hat{A}_{\#}=-d A_{\#}\right\} & & \\
& =\left\{p \in M^{2} \backslash \Sigma_{\#} ; K^{\text {ext }} d \hat{A}=-d A_{\#}\right\} & & \text { (by (c) in Lemma 3.10) } \\
& =\left\{p \in M^{2} \backslash \Sigma_{\#} ; K^{\text {ext }} d A=-d A_{\#}\right\} & & \text { (since } d \hat{A}=d A) \\
& =\left\{p \in M^{2} \backslash \Sigma_{\#} ; K^{\text {ext }}<0\right\} & & \text { (by (d) in Lemma 3.10) } \\
& =\left\{p \in M^{2} ; K^{\text {ext }}<0\right\} . &
\end{array}
$$

Hence we have the conclusion.

Remark 3.13. When $N^{3}(c)=\boldsymbol{R}^{3}$, (3.18) is the classical formula given in 2]. In this case, $S_{\#}^{+}+S_{\#}^{-}$is equal to the total number of cusps appears in the Gauss map $\nu$ of $f$. Romero-Fuster [11] discusses on this number for embedded surfaces. When $N^{3}(c)=S^{3}$ or $H^{3}$, the formula has been also proved in the authors' previous work [16. However, this formula is a generalization of those results in 2] and [16, since 
$N^{3}(c)$ might not be simply connected in our setting. It should be also remarked that the formula (3.18) can be generalized to any compact immersed surfaces in an arbitrary orientable Riemannian 3-manifold: In fact, (2.6) for $\psi_{f}$ is equivalent to the Codazzi equation for the space form and does not hold for a general Riemannian 3-manifold. However, (3.16) still holds without assuming (2.6), as mentioned in Remark 3.4 and the above proof works in this general setting (see [17] for details).

Here, we give two examples satisfying $\chi\left(M^{-}\right)<0$.

Example 3.14. It is well-known that there are embedded triply periodic minimal surfaces. Although the Gauss maps of minimal surfaces only have isolated singular points, if we perturb the surface, we get an immersion $f: M^{2} \rightarrow T^{3}$, where $M^{2}$ is a compact 2-manifold with positive genus. Since minimal surfaces have negative Gaussian curvature, the perturbation $f$ satisfies $\chi\left(M^{-}\right)<0$, and the Gauss map of $f$ must have cusps.

Example 3.15. Similarly, considering the Jorge-Meeks symmetric trinoid with the three ends rounding off to become closed discs, one can get a closed surface in $\boldsymbol{R}^{3}$ as in Fig. 6. Then the resulting surface satisfies $\chi\left(M^{-}\right)<0$, and its Gauss map must have cusps.

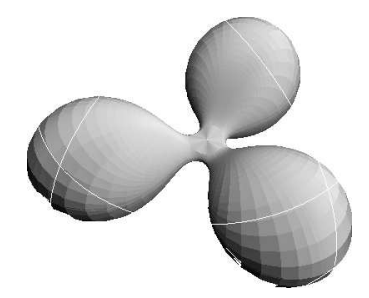

Figure 6. Trinoid with $\chi\left(M^{-}\right)<0$ in Example 3.15

Interchanging the roles of $\varphi$ and $\psi$, we get the following dual assertion in $\boldsymbol{R}^{3}$. Let $f: M^{2} \rightarrow \boldsymbol{R}^{3}$ be a front with unit normal vector $\nu$. Then for each real number $t, f_{t}:=f+t \nu$ is a front with unit normal $\nu$, which is called the parallel front of signed distance $t$ of the front $f$.

Theorem 3.16 (The dual version of Theorem 3.12). Let $f: S^{2} \rightarrow \boldsymbol{R}^{3}$ be a strictly convex surface and $f_{t}(t \in \boldsymbol{R})$ a parallel front of $f$, Assume that the set of singular points of $f_{t}$ consists of cuspidal edges and swallowtails. Then $2 \chi\left(M_{f_{t}}^{-}\right)=S_{f_{t}}^{+}-S_{f_{t}}^{-}$ holds, where $S_{f_{t}}^{+}$(resp. $S_{f_{t}}^{-}$) is the number of positive (resp. negative) swallowtails of $f_{t}$, and $M_{f_{t}}^{-}$is the subset of $M^{2}$ where the Gaussian curvature $K_{t}$ of $f_{t}$ is negative.

Proof. Since $f$ is strictly convex, $\nu: S^{2} \rightarrow S^{2}$ is a diffeomorphism, and then $\Sigma_{\#}$ vanishes. Then, exchanging the roles of $\varphi$ and $\psi$, the proof of Theorem 3.12 implies the conclusion.

Example 3.17. Let $f: S^{2} \rightarrow \boldsymbol{R}^{3}$ be a parametrization of the ellipsoid defined by $\left(x^{2} / 5\right)^{2}+(y / 4)^{2}+z^{2}=1$ and let $\nu$ be its unit normal vector field. Then we can observe that $f_{c}:=f+c \nu$ for $c=11 / 2$ has four negative swallowtails and the Euler number of the set $\left\{K_{c}<0\right\}$ is -2 , where $K_{c}$ is the Gaussian curvature of $f_{c}$, see Fig. 7. 


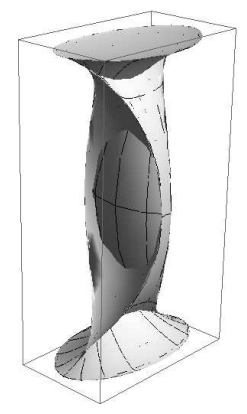

Figure 7. A parallel surface of an ellipsoid with $\chi\left(M^{-}\right)<0$ in Example 3.17 .

On the other hand, as a conclusion of (3.17), we have:

Theorem 3.18. Let $f: M^{2} \rightarrow \boldsymbol{R}^{3}$ be an immersion and $\nu: M^{2} \rightarrow S^{2}$ the Gauss map of $f$. Assume that the singular set $\Sigma_{\#}$ of $\nu$ consists of folds (i.e. $A_{2}$-points) and cusps (i.e. $A_{3}$-points). Then

$$
\int_{\Sigma_{\#}} \kappa_{\#} d \tau_{\#}=\int_{M_{\#}^{-}} K d \hat{A}=\int_{M^{2}} K^{-} d A
$$

holds, where $K^{-}=\min (0, K)$. In particular, the total dual singular curvature (with respect to $\nu) \int_{\Sigma_{\#}} \kappa_{\#} d \tau_{\#}$ is non-positive.

Proof. Since $f$ is an immersion into $\boldsymbol{R}^{3}$ (i.e. $c=0$ ), $M_{\#}^{-}=\left\{p \in M^{2} ; K^{\text {ext }}<0\right\}=$ $\left\{p \in M^{2} ; K<0\right\}$ holds. Then we have

$$
\int_{M_{\#}^{-}} K d A=\int_{M^{2}} K^{-} d A
$$

Here,

$$
\begin{aligned}
\int_{M^{2}} K_{\#} d A_{\#} & =\int_{M_{\#}^{+}} K_{\#} d \hat{A}_{\#}-\int_{M_{\#}^{-}} K_{\#} d \hat{A}_{\#} \\
& =\int_{M_{\#}^{+}} K d \hat{A}-\int_{M_{\#}^{-}} K d \hat{A} \quad \text { (by (a) of Lemma 3.10) } \\
& =\int_{M_{\#}^{+}} K d A-\int_{M_{\#}^{-}} K d A \quad \text { (since } f \text { is an immersion) } \\
& =\int_{M^{2}} K d A-2 \int_{M_{\#}^{-}} K d A \\
& =2 \pi \chi\left(M^{2}\right)-2 \int_{M^{2}} K^{-} d A . \quad(\text { by }(3.20) .
\end{aligned}
$$

Thus, by (3.17), we have the conclusion.

Example 3.19. Consider a rotation of the sine curve

$$
f(u, v):=(u \cos v, u \sin v, \cos u) \quad(0<u<\pi, 0 \leq v \leq 2 \pi)
$$

whose Gauss map has $A_{2}$-singular points of positive singular curvature. This shows that the singular curvature of Gauss maps can take positive values. 
Corollary 3.20. Under the same assumptions as in Theorem 3.18, if there exists a point $p$ satisfying $K(p)<0$ then there exists a fold point such that $\kappa_{\#}$ is negative.

Example 3.21. An embedded Delaunay surface (an unduloid) as a rotationally symmetric periodic surface in $\boldsymbol{R}^{3}$ can be considered as an immersion into a flat space $f: S^{1} \times S^{1} \rightarrow \boldsymbol{R}^{2} \times S^{1}$ which has an annular domain having negative Gaussian curvature. As a consequence, the image of its Gauss map of $f$ has folds of negative singular curvature.

Remark 3.22. Let $f: M^{2} \rightarrow \boldsymbol{R}^{3}$ be an immersion of a compact 2-manifold $M^{2}$. Then the inequality

$$
\frac{1}{2 \pi} \int_{M^{2}}|K| d A \geq \beta_{0}+\beta_{1}+\beta_{2}
$$

is called the Chern-Lashof inequality, where $\beta_{j}(j=0,1,2)$ is the $j$-th Betti number of $M^{2}$. The equality holds if and only if $f$ is tightly immersed in $\boldsymbol{R}^{3}$. On the other hand, by the Gauss-Bonnet formula, it holds that

$$
\frac{1}{2 \pi} \int_{M^{2}} K d A=\beta_{0}-\beta_{1}+\beta_{2} \text {. }
$$

Hence, we have that

$$
-\int_{\Sigma_{\#}} \kappa_{\#} d \tau_{\#}=-\int_{M^{2}} K^{-} d A \geq 2 \pi \beta_{1}
$$

where the equality holds if and only if $f$ is a tight immersion.

Similar to Theorem 3.16 the dual version of Theorem 3.18 holds:

Theorem 3.23. Let $f: S^{2} \rightarrow \boldsymbol{R}^{3}$ be a strictly convex immersion and $f_{t}$ a parallel front of $f$ for $t \in \boldsymbol{R}$. Assume that the set of singular points $\Sigma_{f_{t}}$ of $f_{t}$ consists of cuspidal edges and swallowtails. Then

$$
\int_{\Sigma_{f_{t}}} \kappa_{f_{t}} d \tau_{f_{t}}=\int_{M_{f_{t}^{-}}^{-}} K_{\#} d \hat{A}_{\#}=\int_{M_{f_{t}^{-}}^{-}} K_{t} d \hat{A}_{f_{t}}
$$

holds, where $K_{t}$ is the Gaussian curvature of $f_{t}, M_{f_{t}}^{-} \subset M^{2}$ is the set where $K_{t}<0$, and $\kappa_{f_{t}}$ is the singular curvature of cuspidal edges of $f_{t}$.

Proof. The equality $\int_{\Sigma_{f_{t}}} \kappa_{f_{t}} d \tau_{f_{t}}=\int_{M_{f_{t}}^{-}} K_{\#} d \hat{A}_{\#}$ follows from the proof of Theorem 3.18 by exchanging the roles of $\varphi$ and $\psi$. On the other hand, in the proof of Theorem 3.12 we proved that $M_{\#}^{-}$coincides with the set $\left\{p \in M^{2} ; K^{\operatorname{ext}}(p)<0\right\}$ because of $\varphi_{f}$ has no singular points. In our situation, since $\nu: S^{2} \rightarrow S^{2}$ is a diffeomorphism, $\psi_{f}$ has no singular points. Thus, by exchanging the roles of $\varphi$ and $\psi$, we have that

$$
M_{f_{t}}^{-}=\left\{p \in M^{2} ;\left(K_{t}^{\text {ext }}\right)_{\#}(p)<0\right\}=\left\{p \in M^{2} ; K_{t}^{\text {ext }}(p)<0\right\},
$$

because of (e) in Lemma 3.10 Moreover, by (3.9), we have $K_{t}^{\text {ext }}=K_{t}$ and get the assertion.

Corollary 3.24. Under the same assumptions as in Theorem 3.23, if there exists a point $p$ satisfying $K_{f_{t}}(p)<0$ then there exists a point such that $\kappa_{f_{t}}$ is negative. 
3.4. The case of bounded Gaussian curvature. From now on, we assume that the Gaussian curvature is bounded. As shown below, there are many such surfaces as wave fronts. The following lemma holds.

Lemma 3.25. Let $M^{2}$ be an oriented 2-manifold, $f: M^{2} \rightarrow N^{3}(c)$ a front, and $p$ an $A_{2}$-singular point of $f$. Let $\left(M^{2}, \mathcal{E}_{f},\langle\rangle, D,, \varphi, \psi\right)$ be a front bundle associated to $f$ (cf. Example 2.14). Suppose that there exists a neighborhood $U$ of a $(\varphi$-) singular point $p$ such that $\log \left|K^{\mathrm{ext}}\right|$ is bounded on $U \backslash \Sigma$, where $K^{\mathrm{ext}}$ is the extrinsic Gaussian curvature and $\Sigma$ is the set of singular points of $f$.

Then the following holds on $U$ :

(e) $\Sigma=\Sigma_{\#}$.

(f) By setting $\epsilon:=\operatorname{sgn}\left(\left.K^{\mathrm{ext}}\right|_{U}\right)$, it holds that $M^{+}=M_{\#}^{\epsilon}$ and $\kappa d \tau=$ $\epsilon \kappa_{\#} d \tau_{\#}$, where $\left.K^{\mathrm{ext}}\right|_{U}$ is the restriction of the function $K^{\mathrm{ext}}$ on $U$.

Proof. We fix a positive orthonormal frame field $\left\{\boldsymbol{e}_{1}, \boldsymbol{e}_{2}\right\}$ of $\mathcal{E}_{f}$ and take matrices $G$ and $G_{\#}$ as in (3.10). Since $K^{\text {ext }}$ is bounded on $U$, $\operatorname{det} G_{\#}=0$ holds on $\Sigma \cap U=$ $\{\operatorname{det} G=0\}$ because of (3.13). This implies $\Sigma \subset \Sigma_{\#}$.

On the other hand, by $(\mathrm{e})$ of Lemma 3.10 and the assumptions, $K_{\#}^{\text {ext }}=1 / K^{\text {ext }}$ is also bounded. Then exchanging the roles of $\varphi$ and $\psi$, we have $\Sigma \supset \Sigma_{\#}$. Hence (e) holds.

We shall prove (f) Parametrize the singular set $\Sigma=\Sigma_{\#}$ by a regular curve $\gamma(t)$ on $U$. Since $p$ is an $A_{2}$-point, $\varphi(\dot{\gamma}(t))$ does not vanish, and one can take a $C^{\infty}$-function $\theta=\theta(t)$ such that

$$
\varphi(\dot{\gamma}(t))=|\varphi(\dot{\gamma}(t))|\left(\cos \theta(t) \boldsymbol{e}_{1}+\sin \theta(t) \boldsymbol{e}_{2}\right)
$$

where ${ }^{\cdot}=d / d t$ and $\boldsymbol{e}_{j}=\boldsymbol{e}_{j}(\gamma(t))(j=1,2)$. Then the conormal vector $\boldsymbol{n}(t)$ along $\gamma(t)$ is expressed as

$$
\boldsymbol{n}(t)=-\sin \theta(t) \boldsymbol{e}_{1}+\cos \theta(t) \boldsymbol{e}_{2} .
$$

Let $\omega$ be the connection form of $D$ with respect to the frame $\left\{\boldsymbol{e}_{1}, \boldsymbol{e}_{2}\right\}$ as in (3.3). Then we have

$$
D_{d / d t} \boldsymbol{n}(t)=(\omega(\dot{\gamma}(t))-\dot{\theta}(t)) \frac{\varphi(\dot{\gamma}(t))}{|\varphi(\dot{\gamma}(t))|} .
$$

Substituting this into (2.14), we have

$$
\kappa(t)=-\frac{\operatorname{sgn}(d \lambda(\eta(t)))}{|\varphi(\dot{\gamma}(t))|}(\omega(\dot{\gamma}(t))-\dot{\theta}(t)),
$$

where $\eta(t)$ is a null vector field such that $\{\dot{\gamma}(t), \eta(t)\}$ is positively oriented. Then we have

$$
\kappa(t) d \tau=\kappa(t)|\varphi(\dot{\gamma}(t))| d t=-\operatorname{sgn}(d \lambda(\eta(t)))(\omega(\dot{\gamma}(t))-\dot{\theta}(t)) d t .
$$

Similarly, if we set

$$
\psi(\dot{\gamma}(t))=|\psi(\dot{\gamma}(t))|\left(\cos \theta_{\#}(t) \boldsymbol{e}_{1}+\sin \theta_{\#}(t) \boldsymbol{e}_{2}\right),
$$

we have

$$
\kappa_{\#}(t) d \tau_{\#}=-\operatorname{sgn}\left(d \lambda_{\#}\left(\eta_{\#}(t)\right)\right)\left(\omega(\dot{\gamma}(t))-\dot{\theta}_{\#}(t)\right) d t
$$

where $\eta_{\#}(t)$ is a null vector field with respect to $\psi$ such that $\left\{\dot{\gamma}(t), \eta_{\#}(t)\right\}$ is positively oriented. 
Both of the frame fields $\{\dot{\gamma}(t), \eta(t)\}$ and $\left\{\dot{\gamma}(t), \eta_{\#}(t)\right\}$ are compatible to the orientation of $M^{2}$. Since $\lambda=0$ on $\gamma(t)$, the relations (3.11) and (3.13) yield

$$
\operatorname{sgn} d \lambda(\eta(t))=\operatorname{sgn} d \lambda\left(\eta_{\#}(t)\right)=\left(\operatorname{sgn}\left(\left.K^{\text {ext }}\right|_{U}\right)\right)\left(\operatorname{sgn} d \lambda_{\#}\left(\eta_{\#}(t)\right)\right) .
$$

Since $\epsilon=\operatorname{sgn}\left(\left.K^{\mathrm{ext}}\right|_{U}\right)$, we have

$$
\operatorname{sgn} d \lambda(\eta(t))=\epsilon d \lambda_{\#}\left(\eta_{\#}(t)\right) .
$$

Finally, by [13, Theorem 3.1], the second fundamental form $I I$ vanishes on $\gamma(t)$. Thus by (3.21) and (3.23), we have

$$
\begin{aligned}
0 & =-I I(\dot{\gamma}(t), \dot{\gamma}(t))=-\langle\varphi(\dot{\gamma}(t)), \psi(\dot{\gamma}(t))\rangle \\
& =-|\varphi(\dot{\gamma})||\psi(\dot{\gamma})| \cos \left(\theta(t)-\theta_{\#}(t)\right),
\end{aligned}
$$

and thus $\theta(t)-\theta_{\#}(t)$ is constant. Hence

$$
\dot{\theta}(t)=\dot{\theta}_{\#}(t)
$$

Summing up, by (3.22), (3.24), 3.25) and (3.26), we have (f)

Corollary 3.26. Let $M^{2}$ be a compact oriented 2-manifold, and $f: M^{2} \rightarrow \boldsymbol{R}^{3}$ a front such that $K>0$ and $\log |K|$ is bounded on $M^{2} \backslash \Sigma$. Then the singular curvature at the $A_{2}$-points of the Gauss map of $f$ is always negative.

Example 3.27. We set

$$
f(u, v):=((2-\cos u) \cos v,(2-\cos u) \sin v, u-\sin u) .
$$

which is a rotation of a cycloid. By a straightforward calculation, $K$ is bounded. In particular, the Gauss map of $f$ has a fold of negative singular curvature.

Theorem 3.28. Let $M^{2}$ be a compact oriented 2-manifold, and $f: M^{2} \rightarrow N^{3}(c)$ a front such that $\log \left|K^{\mathrm{ext}}\right|$ is bounded on $M^{2} \backslash \Sigma$. Suppose that the singular sets $\Sigma$ of $\varphi$ and $\Sigma_{\#}(=\Sigma)$ of $\psi$ consist of $A_{2}$-points and $A_{3}$-points. Then

$$
S^{+}-S^{-}=\operatorname{sgn}\left(K^{\mathrm{ext}}\right)\left(S_{\#}^{+}-S_{\#}^{-}\right)
$$

holds, where $S^{+}$(resp. $S^{-}$) is the number of positive (resp. negative) swallowtails of $f$, and $S_{\#}^{+}$(resp. $S_{\#}^{+}$) is the number of positive (resp. negative) $A_{3}$-point of the map $\nu$ induced from the unit normal vector field of $f$ as in Remark 3.9.

Proof. Combining (a) of Lemma 3.10, (3.14), (3.16) and (f) of Lemma 3.25, we have the conclusion.

Example 3.29. Let $f: M^{2} \rightarrow \boldsymbol{R}^{3}$ be an immersion of constant mean curvature $1 / 2$ and $\nu: M^{2} \rightarrow S^{2}\left(\subset \boldsymbol{R}^{3}\right)$ its Gauss map. Then the parallel surface $f_{1}=f+\nu$ is a wave front with constant Gaussian curvature 1. Since the unit normal vector field of $f_{1}$ is also $\nu$, the singular set $\Sigma_{\#}$ of $\nu$ coincides with the set $\left\{K_{f}=0\right\}$, where $K_{f}$ is the Gaussian curvature of $f$. Then by Lemma 3.25, $\Sigma=\Sigma_{\#}=\left\{K_{f}=0\right\}$.

It is well-known that there are many constant mean curvature immersed tori in $\boldsymbol{R}^{3}$. On the other hand, Große-Brauckmann 3] constructed triply periodic surfaces in $\boldsymbol{R}^{3}$ with constant mean curvature $1 / 2$. Such surfaces are compact surfaces in a 3 -dimensional flat torus, and then Theorem 3.28 can be applied for $f+\nu$ of them.

Moreover, for fronts of negative extrinsic curvature, we have the following:

Theorem 3.30. Let $M^{2}$ be a compact oriented 2-manifold, and $f: M^{2} \rightarrow N^{3}(c)$ a front such that $K^{\mathrm{ext}}<0$ and $\log \left|K^{\mathrm{ext}}\right|$ is bounded on $M^{2}$. Then $\chi\left(M^{2}\right)=0$ holds. 
Proof. By Lemma 3.25, we have $\Sigma=\Sigma_{\#}$ and $\kappa d \tau=-\kappa_{\#} d \tau_{\#}$. Then by (3.15) and (3.17), we have

$$
\begin{aligned}
\int_{M^{2}} K d A & =2 \pi \chi\left(M^{2}\right)-2 \int_{\Sigma} \kappa d \tau=2 \pi \chi\left(M^{2}\right)+2 \int_{\Sigma} \kappa_{\#} d \tau_{\#} \\
& =2 \pi \chi\left(M^{2}\right)+\left(2 \pi \chi\left(M^{2}\right)-\int_{M^{2}} K_{\#} d A_{\#}\right) .
\end{aligned}
$$

On the other hand, by (f) in Lemma 3.25 and (a) in Lemma 3.10 we have

$$
\begin{aligned}
\int_{M^{2}} K_{\#} d A_{\#} & =\int_{M_{\#}^{+}} K_{\#} d \hat{A}_{\#}-\int_{M_{\#}^{-}} K_{\#} d \hat{A}_{\#}=\int_{M^{-}} K_{\#} d \hat{A}_{\#}-\int_{M^{+}} K_{\#} d \hat{A}_{\#} \\
& =\int_{M^{-}} K d \hat{A}-\int_{M^{+}} K d \hat{A}=-\int_{M^{2}} K d A .
\end{aligned}
$$

Combining these, we have the conclusion.

Example 3.31. It is well-known that there is a front of constant Gaussian curvature -1 which is diffeomorphic to a torus, that is, Euler number vanishes. Moreover, we can construct an example of a compact front of non-constant negative Gaussian curvature as follows: We set

$$
f(u, v):=((2+\cos u) \cos v,(2+\cos u) \sin v, u-\sin u),
$$

which is another rotation of a cycloid (cf. Example 3.27). One can easily check that $K$ is bounded. Since $f$ is periodic, it can be considered as a torus in a flat space form $\boldsymbol{R}^{2} \times S^{1}$.

Example 3.32. Let $f: M^{2} \rightarrow S^{3}$ be a flat front in the unit 3-sphere, that is, $f$ has vanishing Gaussian curvature on its regular points. Then the unit normal vector field $\nu: M^{2} \rightarrow S^{3}$ also gives a flat fronts. If $M^{2}$ is compact, $f$ is weakly complete in the sense of [6] and $M^{2}$ is orientable (see [6, Section 1]). On the other hand by Theorem 3.30, the Euler characteristic of $M^{2}$ vanishes because $K^{\text {ext }}=-1$ for flat surfaces in $S^{3}$. Thus $M^{2}$ is diffeomorphic to a torus. Moreover the singular set of $f$ coincides with that of $\nu$, and Theorem 3.28 yields that $S^{+}-S^{-}=-\left(S_{\#}^{+}-S_{\#}^{-}\right)$ holds. This identity is not trivial since the set of swallow tails of $f$ is not equal to that of $\nu$. (In fact, $p$ is an $A_{3}$-point, the null direction should be tangential direction of the singular set $\Sigma$. On the other hand, null directions of $\varphi$ and $\psi$ are linearly independent because $f$ is a front.) Several other properties of flat tori in $S^{3}$ as fronts are discussed in [6].

\section{ACKNOWLEDGEMENT}

The authors thank Wayne Rossman for careful reading of the first draft for giving valuable comments. They also thank the referee for valuable comments.

\section{REFERENCES}

[1] V. I. Arnol'd, S. M. Gusein-Zade and A. N. Varchenko, Singularities of Differentiable MAPS, VOL. 1, Monographs in Math. 82, Birkhäuser, 1985.

[2] D. Bleecker and L. Wilson, Stability of Gauss maps, Illinois J. of Math. 22 (1978) 279-289.

[3] K. Große-Brauckmann, Gyroids of constant mean curvature, Experiment. Math. 6 (1997), $33-50$.

[4] S. Izumiya and K. Saji, A mandala of Legendrian dualities for pseudo-spheres of LorentzMinkowski space and "flat" spacelike surfaces, preprint. 
[5] S. Izumiya, K. Saji and M. Takahashi, Horospherical flat surfaces in Hyperbolic 3-space, J. Math. Soc. Japan 62 (2010), 789-849.

[6] Y. Kitagawa and M. Umehara, Extrinsic diameter of immersed flat tori in $S^{3}$, preprint.

[7] M. Kokubu, W. Rossman, K. Saji, M. Umehara and K. Yamada, Singularities of flat fronts in hyperbolic 3-space, Pacific J. of Math. 221 (2005), 303-351.

[8] H. Levine, Mappings of manifolds into the plane, Amer. J. Math. 88 (1966), 357-365.

[9] H. Liu, M. Umehara and K. Yamada, The duality of conformally flat manifolds, to appear in Bulletin of the Brazilian Mathematical Society, arXiv:1001.4569.

[10] J. R. Quine, A global theorem for singularities of maps between oriented 2-manifolds, Trans. Amer. Math. Soc. 236 (1978) 307-314.

[11] M. C. Romero-Fuster, Sphere stratifications and the Gauss map, Proc. Roy. Soc. Edinburgh Sect. A 95 (1983) 115-136.

[12] K. Saji, Criteria for singularities of smooth maps from the plane into the plane and their applications, Hiroshima Math. J. 40 (2010), 229-239.

[13] K. Saji, M. Umehara and K. Yamada, The geometry of fronts, Ann. of Math. 169 (2009) 491-529.

[14] K. Saji, M. Umehara and K. Yamada, Behavior of corank one singular points on wave fronts, Kyushu J. Math. 62 (2008), 259-280.

[15] K. Saji, M. Umehara and K. Yamada, $A_{k}$-singularities of wave fronts, Math. Proc. Camb. Phil. Soc. 146 (2009), 731-746.

[16] K. Saji, M. Umehara and K. Yamada, The duality between singular points and inflection points on wave fronts, Osaka J. Math. 47 (2010), 591-607.

[17] K. Saji, M. Umehara and K. Yamada, Singularities of Blaschke normal maps of convex surfaces, C. R. Acad. Sci. Paris, Ser. I 348 (2010), 665-668

[18] K. Saji, M. Umehara and K. Yamada, $A_{2}$-singularities of hypersurfaces with non-negative sectional curvature in Euclidean space, preprint.

(Saji) Department of Mathematics, Faculty of Education, Gifu University, Yanagido 1-1, Gifu 501-1193, JAPAN

E-mail address: ksaji@gifu-u.ac.jp

(Umehara) Department of Mathematics, Graduate School of Science, Osaka UniverSITY, TOYONAKA, OSAKA 560-0043, JAPAN

E-mail address: umehara@math.sci.osaka-u.ac.jp

(Yamada) Department of Mathematics, Tokyo Institute of Technology, O-okayama, Meguro, TOKYO 152-8550, JAPAN

E-mail address: kotaro@math.titech.ac.jp 